\title{
High-order fluid model for streamer discharges: I. Derivation of model and transport data
}

\author{
S Dujko ${ }^{1,2}$, A H Markosyan ${ }^{1}$, R D White ${ }^{3}$ and U Ebert ${ }^{1,4}$ \\ ${ }^{1}$ Centrum Wiskunde \& Informatica (CWI), PO Box 94079, 1090 GB Amsterdam, The Netherlands \\ ${ }^{2}$ Institute of Physics, University of Belgrade, PO Box 68, 11080 Zemun, Belgrade, Serbia \\ ${ }^{3}$ ARC Centre for Antimatter-Matter Studies, School of Engineering and Physical Sciences, James Cook \\ University, Townsville 4810, Australia \\ ${ }^{4}$ Department of Applied Physics, Eindhoven University of Technology, PO Box 513, 5600 MB \\ Eindhoven, The Netherlands \\ E-mail: S.Dujko@cwi.nl
}

Received 22 February 2013, in final form 10 September 2013

Published 28 October 2013

Online at stacks.iop.org/JPhysD/46/475202

\begin{abstract}
Streamer discharges pose basic problems in plasma physics, as they are very transient, far from equilibrium and have high ionization density gradients; they appear in diverse areas of science and technology. This paper focuses on the derivation of a high-order fluid model for streamers. Using momentum transfer theory, the fluid equations are obtained as velocity moments of the Boltzmann equation; they are closed in the local mean energy approximation and coupled to the Poisson equation for the space charge generated electric field. The high-order tensor in the energy flux equation is approximated by the product of two lower order moments to close the system. The average collision frequencies for momentum and energy transfer in elastic and inelastic collisions for electrons in molecular nitrogen are calculated from a multi-term Boltzmann equation solution. We then discuss, in particular, (1) the correct implementation of transport data in streamer models; (2) the accuracy of the two-term approximation for solving Boltzmann's equation in the context of streamer studies; and (3) the evaluation of the mean-energy-dependent collision rates for electrons required as an input in the high-order fluid model. In the second paper in this sequence, we will discuss the solutions of the high-order fluid model for streamers, based on model and input data derived in this paper.
\end{abstract}

(Some figures may appear in colour only in the online journal)

\section{Introduction}

Streamers are rapidly growing filaments of weakly ionized plasma, whose dynamics are controlled by highly localized space charge regions and steep plasma density gradients. The dynamics of the streamer ionization front are governed by electron dynamics in electric fields above the breakdown value; therefore, the plasma is very far from equilibrium, and the neutral gas stays cold while the ionization front passes.

Streamers occur widely in pulsed discharges, both in nature and in technology. As their size scales with inverse gas density, streamers occur in the limited volumes of plasma technological devices mostly at high gas densities, while planetary atmospheres also can host huge discharges at very low gas densities; so-called sprite discharges [1-3] are streamers existing at altitudes of up to $90 \mathrm{~km}$ in our atmosphere, i.e. at pressures down to well below $10 \mu$ bar. For a cluster issue on streamers, sprites and lightning, we refer to [4] and the 19 original papers therein, discussing common issues of streamer dynamics in atmospheric discharges and plasma technology, including discharge evolution and subsequent chemical reactions. Streamers are a key element in gas processing in so-called corona reactors $[5,6]$ as well as in high-voltage technology [7]; they are used for the treatment of polluted gases [8] and water [9] or for plasma-enhanced vapour deposition [10]. The plasma bullets observed in plasma jets in 
noble gases have now been identified with streamers [11-16]. Studies of breakdown phenomena at atmospheric pressure in short non-uniform air gaps [17] or in microwave fields [18] benefit from related streamer studies. Streamers appear as well in resistive plate chambers used in high-energy physics, where the streamer mode of operation must be carefully controlled and optimized [19-21].

The progress of streamer simulations with fluid models has recently been reviewed in [22]. The main point for this paper is that essentially all these numerical studies are based on the classical fluid model, as we have called it in [23-25]. The classical fluid model for electron density contains a reaction term for impact ionization and attachment, a drift term accounting for electron displacement in the local field, and electron diffusion; the reaction term is typically taken as a function of the local electric field. The structure of this classical model with reaction, drift and diffusion is based on basic symmetry considerations and conservation laws; it has generally emerged as a purely phenomenological model in the course of the past century, though it was also derived later from the Boltzmann equation by Gogolides and Sawin [26].

On the other hand, cross-sections for the collisions of electrons with molecules have become available with growing accuracy [27-31]. They are the input either for the Boltzmann equation or for Monte Carlo models. These microscopic particle models have not yet been appropriately linked with the much older phenomenological fluid models. This becomes evident when comparing their solutions. A negative streamer ionization front in nitrogen represented by a Monte Carlo model cannot appropriately be described by a classical fluid model even if the transport and reaction coefficients for the fluid model are derived from the Monte Carlo model [32]. In [32], the coefficients for the fluid model were derived as so-called bulk coefficients; therefore, in this model the electron swarms evolve correctly, and ionization fronts in a given electric field propagate with the correct velocity, as they are so-called pulled fronts [32]. However, the electron density behind the ionization front is very low. As we will see in section 3 of this paper, a classical fluid model evaluated with flux coefficients gives better ionization densities behind the front, but very low front velocities.

A phenomenological extension of the classical fluid model with a gradient expansion considerably improves the approximation of the microscopic electron dynamics [33, 34], and it cures the deficiency of the local field approximation by including a dependence of the impact ionization rate on the local electron density gradient [23]. That the extended fluid model matches the Monte Carlo model well up to the moment of streamer branching, can also be seen in a recent comparison of three-dimensional model simulations in [25]. On the other hand, the model is still based on a local field approximation, therefore electron energies in the streamer interior are modelled erroneously as relaxing instantaneously to the low field in the streamer interior.

A full simulation by Monte Carlo models would deliver the physically most reliable results, but this is computationally extremely costly. As a compromise between accuracy and efficiency, hybrid methods [23-25,35] have been developed in the past years to track the fast and energetic processes in the ionization front with a particle model, and to model the many electrons at lower fields in the streamer interior with a fluid model. Fluid models are also the most efficient for the long time evolution in the streamer interior where slow chemical reactions and a slow thermalization of the deposited energy set in.

These demands on fast and quantitative streamer simulations ask for the development of a fluid model that accurately incorporates the microscopic electron dynamics contained in Boltzmann or Monte Carlo models. We will follow here the strategy to derive such a model not from phenomenological considerations, but through a systematic derivation from the Boltzmann equation.

This paper is the first in an ongoing investigation of high-order fluid models for streamer discharges; it is focused on the derivation of first- and higher order fluid models and on the derivation and correct implementation of transport data in these models. Section 2 describes the derivation of models of different order. The starting point is the set of balance equations obtained as velocity moments of the Boltzmann equation. Then the derivation of the classical first-order model is briefly described, and previous approaches to higher order models are summarized. In section 2.4, our new high-order fluid model is derived in a systematic manner. The balance/moment equations are closed after the balance equation for the energy flux. This is done by approximating the high-order tensors in the energy flux balance equation by a product of two lower moments while the collision transfer terms are evaluated using momentum transfer theory. In section 3, electron transport properties as an input in fluid models are calculated using a multi-term theory for solving the Boltzmann equation [36,37]. We pay particular attention to the accuracy of the calculation and the proper use in both the first- and high-order fluid models. The results of our multi-term solution of Boltzmann's equation are compared with those obtained by the publicly available Boltzmann solver BOLSIG+ developed by Hagelaar and Pitchford [38] for electrons in molecular nitrogen. BOLSIG+ is a popular Boltzmann solver based on a classical two-term theory, so we have been motivated to check its accuracy and integrity against the advanced and highly sophisticated multi-term Boltzmann solver developed by the group from the James Cook University and their associates $[36,37,39,40]$. In section 4 , we give the results for negative planar fronts obtained with the firstorder, model with particular emphasis upon the consistent implementation of transport data. It should be noted that a full 3D streamer channel is characterized by a high field enhancement at its growing tip. This field enhancement is created by a thin space charge layer around its ionized interior that in turn largely suppresses the interior field. The thickness of the space charge layer is much smaller than its radius of curvature $[23,24,32,41,42]$. Therefore, when zooming into the front structure, the curvature can be neglected. Modelling this front structure is the largest challenge for a fluid model and therefore this is what we focus on in section 4 . The results for various streamer properties obtained with different types of input data are compared. A thorough analysis of 
numerical streamer front solutions with our high-order model, and a comparison with Monte Carlo results is contained in an accompanying second paper [43].

\section{Derivation of first- and higher order fluid models}

\subsection{General considerations: Boltzmann equation and moment equations}

Our starting point is the Boltzmann equation for charged particles in an electric field $\boldsymbol{E}$

$$
\partial_{t} f_{i}+c \cdot \nabla f_{i}+\frac{e_{i}}{m_{i}} \boldsymbol{E} \cdot \nabla_{c} f_{i}=-J\left(f_{i}, f_{0}\right) .
$$

Here $f_{i}(\boldsymbol{r}, \boldsymbol{c}, t)$ is the distribution function in phase space at the position $\boldsymbol{r}$ and velocity $\boldsymbol{c}$ for each charged component $i$, $\nabla$ is the differential operator with respect to space $r$ and $\nabla_{c}$ with respect to velocity $c, e_{i}$ and $m_{i}$ are charge and mass of species $i$, and $t$ is time. The right-hand side of equation (1), $J\left(f_{i}, f_{0}\right)$, describes the collisions of charged particles with neutral molecules, accounting for elastic, inelastic and nonconservative (e.g. ionizing or attaching) collisions, and $f_{0}$ is the velocity distribution function of the neutral gas (usually taken to be Maxwellian at temperature $T_{\mathrm{g}}$ ).

Streamer discharges have a characteristic non-linear coupling between densities of charged particles and electric field. The space charge modifies the field, and the field determines the drift, diffusion and rate coefficients. The electric field has to be calculated self-consistently with Poisson's equation

$$
\nabla \cdot \boldsymbol{E}=\frac{1}{\epsilon_{0}} \sum_{i} e_{i} n_{i}(\boldsymbol{r}, t),
$$

where $\epsilon_{0}$ is the dielectric constant, and $e_{i}$ and $n_{i}$ are the charges and densities of species $i$ that can be electrons and ions. As ions are much heavier than electrons, their motion is typically neglected within the ionization front, and the analysis focuses on the electron evolution. Thus, in what follows we suppress the charged particle index $i$ in equation (1) and focus on the electron dynamics.

After solving equations (1) and (2), quantities of physical interest could be obtained as velocity 'moments' of the distribution functions, starting with the number density

$$
n(\boldsymbol{r}, t)=\int f(\boldsymbol{r}, \boldsymbol{c}, t) \mathrm{d} \boldsymbol{c},
$$

followed by higher order quantities

$$
\langle\phi(\boldsymbol{c})\rangle=\frac{1}{n(\boldsymbol{r}, t)} \int \phi(\boldsymbol{c}) f(\boldsymbol{r}, \boldsymbol{c}, t) \mathrm{d} \boldsymbol{c},
$$

with $\phi(c)=m c, \frac{1}{2} m c^{2}, \ldots$ furnishing the average velocity $\boldsymbol{v}=\langle\boldsymbol{c}\rangle$, average energy $\varepsilon=\left\langle\frac{1}{2} m c^{2}\right\rangle$ and so on.

Equations (1) and (2) represent a non-linear coupled system of partial differential equations in six-dimensional phase space and in time, with a complicated collision operator $J$. For a complete problem description, appropriate initial and boundary conditions on $f(\boldsymbol{r}, \boldsymbol{c}, t)$ in phase space must be implemented.

For streamers, it is very difficult to solve equations (1) and (2) due to the large gradients of electric fields and of charged particle densities at their fronts. Moreover, the streamers create a self-consistent field enhancement at their tips, which allow them to penetrate into regions where the background field is too low for efficient ionization processes to take place. From a kinetic theory point of view, this is a non-stationary, non-hydrodynamic and non-linear problem where space and time should be treated on an equal footing with velocity in equation (1). Clearly, the numerical solution of equations (1) and (2) for the full streamer problem is a formidable task and a fluid equation treatment is more tractable. In a fluid approach, the problem of solving the Boltzmann equation for $f$ in phase space is replaced by a set of low-order approximate (velocity) moment equations of $f[36,44,45]$.

Fluid equations may be derived either directly as moments of (1) or from first principles using physical arguments. Following the first approach, the set of moment/balance equations can be found by multiplying (1) by $\phi(c)$ and integrating over all velocities:

$\partial_{t}(n\langle\phi(\boldsymbol{c})\rangle)+\nabla \cdot(n\langle\boldsymbol{c} \phi(\boldsymbol{c})\rangle)-n \frac{e}{m} \boldsymbol{E} \cdot\left\langle\nabla_{c} \phi(\boldsymbol{c})\right\rangle=C_{\phi}$,

where \langle\rangle represents the average over the velocity $c$ of the charged particles, and $C_{\phi}$ is the collision term in the balance equation:

$$
C_{\phi}=-\int \phi(c) J(f) \mathrm{d} c .
$$

To derive the term $\left\langle\nabla_{c} \phi(c)\right\rangle$ with its negative sign, a partial integration over $c$ has been performed.

If one now takes $\phi(c)$ consecutively equal to $1, m c, \frac{1}{2} m c^{2}$ and $\frac{1}{2} m c^{2} c$, etc, equation (5) generates an infinite series of equations, a full solution of which would be equivalent to calculating $f$ itself. In practice, one truncates after a certain moment equation; in this process obviously some information of the Boltzmann equation is lost. Therefore, we now discuss the derivation of first- and second-order fluid models with their truncations of the moment equations, and then we derive systematically our new high-order fluid model.

\subsection{The first-order or classical fluid model}

First, we derive the classical fluid model as an approximation from the Boltzmann equation. We use continuity and momentum balance equation, i.e. $\phi(c)=1$ and $m c$, and truncate the set (5) at the momentum balance equation,

$$
\begin{gathered}
\partial_{t} n+\nabla \cdot(n \boldsymbol{v})=C_{1}, \\
\partial_{t}(n m \boldsymbol{v})+\nabla \cdot(n m\langle\boldsymbol{c c}\rangle)-n e \boldsymbol{E}=C_{m c},
\end{gathered}
$$

where $\boldsymbol{v}=\langle\boldsymbol{c}\rangle$ is the average local electron velocity. Now the velocities are decomposed into an average velocity $\boldsymbol{v}$ plus random velocities $\boldsymbol{c}-\boldsymbol{v}$ with zero mean $(\langle\boldsymbol{c}-\boldsymbol{v}\rangle=0)$. On introducing the pressure tensor

$$
\boldsymbol{P}=n m\langle(\boldsymbol{c}-\boldsymbol{v})(\boldsymbol{c}-\boldsymbol{v})\rangle,
$$


equation (8) becomes

$$
\partial_{t}(n m \boldsymbol{v})+\nabla \cdot(n m \boldsymbol{v} \boldsymbol{v})+\nabla \cdot \boldsymbol{P}-n e \boldsymbol{E}=C_{m c},
$$

where the following identity was used

$$
\begin{aligned}
\nabla \cdot & (n m\langle\boldsymbol{c c}\rangle)=\nabla \cdot[n m(\boldsymbol{v} \boldsymbol{v}+\boldsymbol{v}\langle\boldsymbol{c}-\boldsymbol{v}\rangle+\langle\boldsymbol{c}-\boldsymbol{v}\rangle \boldsymbol{v} \\
& +\langle(\boldsymbol{c}-\boldsymbol{v})(\boldsymbol{c}-\boldsymbol{v})\rangle)]=\nabla \cdot(n m \boldsymbol{v} \boldsymbol{v}) \\
& +\nabla \cdot(n m\langle(\boldsymbol{c}-\boldsymbol{v})(\boldsymbol{c}-\boldsymbol{v})\rangle)] .
\end{aligned}
$$

The second term on the left-hand side of (10) can be expanded as

$$
\nabla \cdot(n m \boldsymbol{v} \boldsymbol{v})=n m(\boldsymbol{v} \cdot \nabla) \boldsymbol{v}+\boldsymbol{v}[\nabla \cdot(n m \boldsymbol{v})] .
$$

Now we substitute (12) into (10), use the continuity equation (7) and introduce the convective time derivative

$$
\frac{\mathrm{d}}{\mathrm{d} t}=\partial_{t}+\boldsymbol{v} \cdot \nabla
$$

which measures the rate of change in a reference frame moving with the mean drift velocity $\boldsymbol{v}$ of the electrons. The momentum balance equation then obtains the form

$$
n m \frac{\mathrm{d} \boldsymbol{v}}{\mathrm{d} t}=n e \boldsymbol{E}-\nabla \cdot \boldsymbol{P}+C_{m c}-m \boldsymbol{v} C_{1} .
$$

The physical interpretation of this equation is straightforward: the rate of change of the mean electron velocity is due to the force of the electric field plus forces due to the pressure of the electron fluid itself and due to internal forces associated with the collisional interactions with a large number of neutral gas molecules. It should be emphasized, however, that the form with the convective derivative $\mathrm{d} / \mathrm{d} t$ is not useful for the analysis of a full streamer problem where local fields and therefore local mean electron velocities $\boldsymbol{v}$ vary largely in space and time.

Because the system has been truncated, the yet unspecified tensor $\boldsymbol{P}$ (9) of electron pressure appears on the right-hand side of (14), and a closure assumption needs to be found. If the distribution of random velocities is close to isotropic, the diagonal terms of $\boldsymbol{P}$ are equal and given by the scalar kinetic pressure $p$,

$$
\boldsymbol{P} \approx p \boldsymbol{I}=n k T,
$$

where $\boldsymbol{I}$ is the unity tensor, $k$ is the Boltzmann constant and $T$ is the electron temperature. It should be emphasized here that the isotropy of the velocity fluctuations and of the pressure is a strong assumption at the streamer tip where the electric fields are high and strong pressure gradients exist. In this streamer region, the random spread of electrons along the field direction can differ significantly from the perpendicular direction.

The next simplifying assumption concerns the collision terms. An expression often used for momentum transfer by collision is

$$
C_{m c}=-n m v_{\mathrm{eff}} \boldsymbol{v},
$$

which assumes that the force per unit volume exerted on the electrons due to collisions with neutral molecules is proportional to the average electron velocity. The proportionality constant is called the effective momentum transfer collision frequency; it accounts for momentum exchange in elastic and inelastic collisions. With this simplifying assumption and neglecting the transfer of momentum in non-conservative collisions ${ }^{5}$ relative to other momentum transfer collisions (which is usually a good approximation), the momentum balance equation (14) becomes

$$
n m \frac{\mathrm{d} \boldsymbol{v}}{\mathrm{d} t}=n e \boldsymbol{E}-\nabla p-n m v_{\mathrm{eff}} \boldsymbol{v} .
$$

If the rate of momentum change $\left(\mathrm{d}_{t} \boldsymbol{v}\right) / \boldsymbol{v}$ is smaller than the rate of momentum transfer $\nu_{\text {eff }}$, and if the gradients in electron energy can be neglected, one gets the following expression for the average flux of the electrons:

$$
\boldsymbol{\Gamma}=n \boldsymbol{v}=n \mu \boldsymbol{E}-D \nabla n,
$$

where mobility and diffusion constant are given by

$$
\mu=\frac{e}{m v_{\mathrm{eff}}}, \quad D=\frac{k T}{m v_{\mathrm{eff}}},
$$

if the system is close to equilibrium. In this case the NernstTownsend-Einstein relation

$$
\frac{D}{\mu}=\frac{k T}{e}
$$

is valid. The steady-state form of equation (17) is an acceptable approximation because the effective time constant for momentum transfer $\frac{1}{v_{\text {eff }}}$ at atmospheric pressure is generally much less than the time scale on which the local electric field varies within a streamer $[32,46]$.

Further from equilibrium as in the head of the streamer where the electric fields are high, the approximation (15) is not valid as the velocity fluctuations of the electrons are clearly anisotropic. Let us consider once more the special case where the average velocity of electrons is stationary. From equation (14) and neglecting momentum transfer in non-conservative collisions, we obtain

$$
m n v_{\mathrm{eff}} \boldsymbol{v}=e n \boldsymbol{E}-\nabla \cdot \boldsymbol{P},
$$

and as a result for the flux we have

$$
\boldsymbol{\Gamma}=n \mu \boldsymbol{E}-\frac{1}{v_{\mathrm{eff}}} \nabla \cdot[n\langle(\boldsymbol{c}-\boldsymbol{v})(\boldsymbol{c}-\boldsymbol{v})\rangle] .
$$

Comparing the last equation and equation (18), it is obvious that quantity

$$
D^{\star}=\frac{\langle(c-v)(c-v)\rangle}{v_{\mathrm{eff}}}
$$

is the reminisce of the diffusion tensor often used in the drift-diffusion approximation instead of the diffusion constant. However, the physical interpretation of this quantity is not $a$ priori clear. Although this quantity assumes the anisotropic nature of the temperature tensor, it reduces to the diffusion constant $D$ when the effective momentum transfer collision frequency $v_{\text {eff }}$ is independent of the electron energy. For an energy-dependent effective momentum transfer collision frequency, the straightforward generalization of the Einstein 5 Non-conservative collisions do not conserve particle numbers as they
account for ionization, attachment or recombination reactions. 
relation for the diffusion constant (19) to the diffusion tensor $\boldsymbol{D}$ or application of $\boldsymbol{D}^{\star}$ in the equation for the average flux (18) is misleading and the reader is referred to [47-49] where the so-called generalized Einstein relation was introduced using the momentum and energy balance equations. However, the generalization of the diffusion constant $D$ appearing in (18) to diffusion tensor $D$ is a very welcome step in fluid modelling of streamer discharges due to the often strong anisotropic nature of the diffusion tensor for certain gases and due to deviations of the electron velocity distribution function from a Maxwellian distribution of velocities in different spatial regions of the streamers.

When the above approximation for the particle flux is inserted into equation (7), we get the well-known reaction drift-diffusion expression for the charged particle motion in the discharge:

$$
\partial_{t} n+\nabla \cdot(\mu(\boldsymbol{E}) \boldsymbol{E} n-\boldsymbol{D}(\boldsymbol{E}) \cdot \nabla n)=C_{1} .
$$

Originally, this approximation was not derived from the Boltzmann equation, but derived on purely phenomenological grounds. It is clear that the equation must have the structure of a continuity equation with a source term for electron generation and loss. The parametric dependence of the source term is open at this point; within the classical model the source term is assumed to depend on local particle densities and on the local electric field. The second approximation concerns the electron velocity $\boldsymbol{v}$. On a time scale much larger than the collision frequency, the electron motion is overdamped, so the electrons must drift against the direction of the electric field according to the second term in (24). The stochastic and undirected motion is modelled in an ad hoc manner through the diffusion term; the fact that the diffusion correction is added to the drift term and not included in any other functional manner is not a priori clear and can actually be deduced from the above analysis.

In conclusion, the lowest level of fluid approximation, also called classical model or first-order model, is given by the equations

$$
\begin{aligned}
\frac{\partial n}{\partial t}=\nabla \cdot(\boldsymbol{D} \cdot \nabla n)+\nabla & (\mu n \boldsymbol{E})+n\left(v_{\mathrm{I}}-v_{\mathrm{A}}\right), \\
\frac{\partial n_{\mathrm{p}}}{\partial t} & =n v_{\mathrm{I}}, \\
\frac{\partial n_{\mathrm{n}}}{\partial t} & =n v_{\mathrm{A}},
\end{aligned}
$$

coupled to the Poisson equation for the electric field,

$$
\nabla^{2} \phi=\frac{e}{\epsilon_{0}}\left(n-n_{\mathrm{p}}+n_{\mathrm{n}}\right), \quad \boldsymbol{E}=-\nabla \phi .
$$

Here $n_{\mathrm{p}}$ and $n_{\mathrm{n}}$ are positive and negative ion densities, while $v_{\mathrm{I}}$ and $v_{\mathrm{A}}$ are the ionization and attachment collision frequencies due to electron-molecule collisions, and $\phi$ is the electric potential. The numerical solution of the system (25)-(28) requires the transport properties $\mu, D, v_{\mathrm{I}}$ and $v_{\mathrm{A}}$ as a function of the local electric field for the gas in question. The derivation and implementation of transport data are discussed in section 3 . The numerical solutions of planar streamer fronts with these transport data are discussed in section 4.

\subsection{Second-order models including the energy balance} equation

We now turn to models that include the second velocity moment of the Boltzmann equation, i.e. the energy balance equation. We will see that the closure of this equation is not a straightforward process, and we will discuss some approximations made in the literature. The results of a second-order model and of our high-order model for planar streamer fronts will be compared in our second paper [43].

The first important steps beyond first-order fluid models of streamer discharges, to our knowledge, were carried out by Abbas and Bayle [50,51], and by Bayle and Cornebois [52]. They employed a second-order model, which involves the energy balance equation to explore the zone at the streamer tip where the electron energy is not determined anymore by the local electric field only. However, as pointed out by Kanzari et al [46], the accuracy of their model was restricted by the drastic assumptions taken in their energy balance equation. They evaluated the source term in the energy balance equation and corresponding averages by assuming a Maxwellian distribution for the electrons. Guo and $\mathrm{Wu}$ [53] have developed a more sophisticated second-order model in which the Langevin theory was used to simplify the collision source terms with a priori knowledge of the relaxation times of electron energy and momentum. Kanzari et al [46] have made an important step further by calculating the source term in the energy equation in a more consistent way where the ad hoc assumptions for the distribution function were avoided. A similar approach was later used by Eichwald et al [54] in their simulations of the streamer dynamics and of the radical formation in a pulsed corona discharge used for flue gas control. Their results showed that the average electron velocity in the streamer head is largely overestimated by the classical first-order model. As a consequence, electron density and radical density in the ionized channel were up to $50 \%$ higher than with the second-order model. The salient feature of their theory is the fact that the heat flux term in the energy balance equation was explicitly neglected, but this, unfortunately, is of questionable accuracy. We will illustrate in streamer simulations in our second paper [43] that the energy flux plays an important role in determining the streamer behaviour. Thus, particular care should be taken with the closure through specification of the energy flux. Even for charged particle swarms under non-hydrodynamic conditions one must be careful how to specify the heat flux vector $[36,44,45,55]$. We now discuss how the fluid equations should be closed for streamers, while we stress that the method itself is applicable to a much wider range of phenomena.

\subsection{The high-order fluid model}

We now derive a fluid model including the energy flux equation, i.e. we truncate at the moment equation with the third power of velocity. We will argue that the energy flux equation is crucial for the success of the fluid model for streamers. Inserting different moments of $c$ into equation (5), one finds

$\frac{\partial n}{\partial t}+\nabla \cdot n \boldsymbol{v}=C_{1}$, 


$$
\begin{aligned}
& \frac{\partial}{\partial t}(n m \boldsymbol{v})+\nabla \cdot(n m\langle\boldsymbol{c c}\rangle)-n e \boldsymbol{E}=C_{m c}, \\
& \frac{\partial}{\partial t}\left(n\left\langle\frac{1}{2} m c^{2}\right\rangle\right)+\nabla \cdot\left(n\left\langle\frac{1}{2} m c^{2} c\right\rangle\right)-n e \boldsymbol{E} \cdot \boldsymbol{v}=C_{\frac{1}{2} m c^{2}}, \\
& \frac{\partial}{\partial t}\left(n\left\langle\frac{1}{2} m c^{2} \boldsymbol{c}\right\rangle\right)+\nabla \cdot\left(n\left\langle\frac{1}{2} m c^{2} c c\right\rangle\right) \\
& \quad-n e \boldsymbol{E} \cdot\left\langle\frac{\partial}{\partial \boldsymbol{c}}\left(\frac{1}{2} m c^{2} \boldsymbol{c}\right)\right\rangle=C_{\frac{1}{2} m c^{2} \boldsymbol{c} .}
\end{aligned}
$$

Different forms of these equations that some readers might be familiar with are given in the appendix. It will be shown in section 2.4.3 that the explicit form of the high-order tensors appearing in the divergence term of the energy flux equation (32) is not required.

This system of equations is exact at this stage, but not very useful and the reason is twofold. First, there are more unknowns than equations, the familiar problem of closure. The set of fluid equations can be increased to an arbitrary size merely by taking higher velocity moments of the Boltzmann equation. Second, the definition of the system requires the respective collision terms $C_{\phi}$.

\subsubsection{Collision processes. In contrast to Monte Carlo} simulations or kinetic equations in which cross-sections for charged particle scattering enter into the calculations in a fairly clear way, in fluid equations the collisions are treated in a variety of ways, which are not necessarily consistent with the data itself and/or with the system under consideration. There are many examples that illustrate this issue and the reader is referred to $[36,44,45]$ for a detailed discussion. In the context of streamer and breakdown studies beyond first-order fluid models, it has become common practice to evaluate the collision terms and averages by assuming some particular form of the velocity distribution function, usually Maxwellian [26, 52, 53, 56-58].

Let us consider the form of the collision terms in the balance equations (29)-(32). In this work, we characterize the elastic, inelastic and non-conservative collisions by corresponding average collision frequencies. A collision frequency $v\left(v_{\mathrm{r}}\right)$ for collisions between charged particles and gas molecules is related to the cross-section $\sigma\left(v_{\mathrm{r}}\right)$ characterizing the process by

$$
v\left(v_{\mathrm{r}}\right)=n_{\mathrm{g}} v_{\mathrm{r}} \sigma\left(v_{\mathrm{r}}\right)
$$

where $\boldsymbol{v}_{\mathrm{r}}$ is the relative velocity and $n_{\mathrm{g}}$ is the number density of the neutral gas. Furthermore, we deal with weakly ionized systems where the interactions of charged particles with one another is negligible.

Our calculation takes into account that the charged particles can lose energy and momentum even in elastic collisions with the gas molecules as the finite mass and the thermal energy of the molecules are taken into account. The momentum exchange in inelastic collisions is included and $v_{m}=v_{m}\left(v_{\mathrm{r}}\right)$ denotes the total momentum transfer collision frequency while $v_{\alpha}$ and $v_{\alpha}^{(s)}$ are inelastic and superelastic collision frequencies for the inelastic channel $\alpha$. The total collision frequencies for attachment and ionization are denoted by $v_{\mathrm{A}}=v_{\mathrm{A}}\left(v_{\mathrm{r}}\right)$ and $v_{\mathrm{I}}=v_{\mathrm{I}}\left(v_{\mathrm{r}}\right)$, respectively. We consider only single ionization with ionization energy $\epsilon_{\mathrm{I}}$, but the resulting ion can be left in any one of its internal excited states, characterized by an excitation energy $\Delta \epsilon_{\mathrm{I}}^{(i)}$ and a collision frequency $v_{\mathrm{I}}^{(i)}$.

2.4.2. Momentum transfer theory. The momentum transfer theory has a long history in kinetic theory of gases and has proved very successful for describing charged particle transport in gases under the influence of electric and magnetic fields. It is discussed comprehensively in the textbook of Mason and McDaniel [48] and in [36, 44, 45, 49, 59-61]. The main ideas can be briefly summarized as follows:

(1) We need to express the average collision frequencies as a function of the mean energy in the centre-of-mass reference frame. Thus, we replace the variables

$$
v_{\mathrm{r}} \rightarrow \varepsilon=\frac{1}{2} \mu_{\mathrm{r}} v_{\mathrm{r}}^{2}
$$

in expressions for collisional frequencies

$$
v=v\left(v_{\mathrm{r}}\right) \rightarrow \widetilde{v}=\widetilde{v}(\varepsilon)
$$

where $\varepsilon$ is the energy measured in the centre-of-mass reference frame and $\mu_{\mathrm{r}}$ is the reduced mass.

(2) If we assume that the dominant contributions to the averages come from regions near the mean energy $\bar{\varepsilon}$, and that $v(\varepsilon)$ varies sufficiently slowly with $\varepsilon$, then a Taylor expansion

$$
\widetilde{v}(\varepsilon)=\widetilde{v}(\bar{\varepsilon})+\widetilde{v}^{\prime}(\bar{\varepsilon})(\varepsilon-\bar{\varepsilon})+\cdots
$$

is expected to be a reasonable approximation. For conservative collisional processes such as elastic and inelastic scattering, only the first term of the expansion (36) is considered. However, when energy-dependent non-conservative processes such as ionization and electron attachment are operative then the derivative term in (36) becomes the leading term and must be included.

(3) Using the momentum transfer approximation, the balance equations (29)-(32) get the form

$$
\begin{aligned}
& \frac{\partial n}{\partial t}+\nabla \cdot n \boldsymbol{v}=-n\left(\widetilde{v}_{\mathrm{A}}-\widetilde{v}_{\mathrm{I}}\right), \\
& \frac{\partial}{\partial t}(m n \boldsymbol{v})+\nabla \cdot(m n\langle\boldsymbol{c c}\rangle)-n e \boldsymbol{E} \\
& =-\mu_{\mathrm{r}} n \widetilde{v}_{m} \boldsymbol{v}-m n \boldsymbol{v}\left[\widetilde{v}_{\mathrm{I}}+\zeta \widetilde{v}_{\mathrm{A}}^{\prime}\right], \\
& \frac{\partial}{\partial t}(n \varepsilon)+\nabla \cdot(n \boldsymbol{\xi})-n e \boldsymbol{E} \cdot \boldsymbol{v} \\
& =-n \widetilde{v}_{e}\left[\left(\varepsilon-\frac{3}{2} k T_{0}\right)+\Omega(\bar{\varepsilon})\right]-n \varepsilon \widetilde{v}_{\mathrm{A}}, \\
& \frac{\partial}{\partial t}(n \boldsymbol{\xi})+\nabla \cdot\left(n\left\langle\frac{1}{2} m c^{2} c c\right\rangle\right)-n e \boldsymbol{E} \cdot\left\langle\frac{\partial}{\partial \boldsymbol{c}}\left(\frac{1}{2} m c^{2} c\right)\right\rangle \\
& =-n \widetilde{v}_{m} \boldsymbol{\xi},
\end{aligned}
$$


where $\varepsilon$ is the average electron energy, $\boldsymbol{\xi}$ is the average electron energy flux and $m_{0}$ is the mass of gas molecules. $\zeta$ is given by $[49,60]$

$$
\zeta=\frac{2}{3} \frac{m_{0}}{m+m_{0}}\left(\frac{1}{2} m\left\langle c^{2}\right\rangle-\frac{1}{2} m|\boldsymbol{v}|^{2}\right) .
$$

The average collision frequencies for momentum and energy transfer

$$
\begin{aligned}
& \widetilde{v}_{m}(\bar{\varepsilon})=n_{\mathrm{g}} \sqrt{\frac{2 \varepsilon}{\mu_{\mathrm{r}}}} \sigma_{m}(\bar{\varepsilon}), \\
& \widetilde{v}_{e}(\bar{\varepsilon})=\frac{2 \mu_{\mathrm{r}}}{m+m_{0}} \widetilde{v}_{m}(\bar{\varepsilon}),
\end{aligned}
$$

are prescribed functions of the mean energy in the centreof-mass frame

$$
\bar{\varepsilon}=\frac{m_{0} \varepsilon+m \frac{3}{2} k T_{0}}{m+m_{0}},
$$

where $k$ is Boltzmann's constant and $T_{0}$ is the neutral gas temperature. The term $\Omega$ represents the average energy lost in one energy relaxation time $\widetilde{v}_{e}^{-1}$, through non-elastic processes and is given by [36]

$\Omega(\bar{\varepsilon})=\frac{m_{0}}{m+m_{0}} \sum_{\alpha} \frac{\left(\widetilde{v}_{\alpha}-\widetilde{v}_{\alpha}^{(s)}\right)}{\widetilde{v}_{e}} \epsilon_{\alpha}+\sum_{i} \frac{\widetilde{v}_{\mathrm{I}}^{(i)}}{\widetilde{v}_{e}} \Delta \epsilon_{\mathrm{I}}^{(i)}$.

The inelastic channels $\alpha$ are governed by threshold energies $\epsilon_{\alpha}$ and collision frequencies for inelastic and superelastic processes $\widetilde{v}_{\alpha}$ and $\widetilde{v}_{\alpha}^{(s)}$, respectively. All collision frequencies describing the inelastic, nonconservative and superelastic collisions appearing in equations (37)-(40) are functions of $\bar{\varepsilon}$. It should be emphasized that the equations of continuity (37), of momentum balance (38) and of energy balance (39) are valid for charged particles of arbitrary mass while the energy flux equation (40) is obtained in the approximation of $m / m_{0} \ll 1$. High-order corrections in momentum transfer theory corrections (e.g. high-order terms in the Taylor expansion (36)) could be added on the right-hand side of the system (37)-(40) if desired, without in any way changing the generality of the physical arguments associated with the closure assumptions presented below.

\subsubsection{Solution regimes and closure assumptions. The} closure of the system of equations (37)-(40) requires approximations or assumptions on the form of the pressure tensor. The standard approximation for light particles such as electrons is that the pressure tensor can be taken as a scalar at the fluid level of approximation [36, 44, 45, 49]. This means that, $\bar{\varepsilon} \approx \varepsilon \gg \frac{1}{2} m v^{2}$, and the pressure tensor simplifies to

$$
\boldsymbol{P}=n k \boldsymbol{T} \approx \frac{2}{3} n \varepsilon \boldsymbol{I},
$$

where $\boldsymbol{T}$ is the so-called temperature tensor that characterizes energy fluctuations even if the system is not in thermal equilibrium. This form of the pressure tensor was employed in all previous swarm-oriented studies $[36,44,45,49,55,59$, $60]$, as well as in the recent fluid models of streamer discharges $[46,54]$. However, if the velocity distribution significantly deviates from isotropy in velocity space, then this approximation is problematic. For ions, the distribution function in velocity space is always anisotropic (even if elastic collisions between ions and molecules are predominant) and hence any assumption on an isotropic pressure tensor is wrong for ions. This problem can be avoided by considering the temperature tensor balance equation but this in turn contains further unknowns. The reader is referred to [61] for how to treat charged particle swarms under spatially homogeneous hydrodynamic conditions. In streamer studies, the ions are usually considered as immobile or they are modelled by a reaction-drift-diffusion and local field approximation, which is a reasonable approximation on the time scale on which a streamer ionization front passes a given point in space.

The next step in the closure of the system of equations (37)-(40) concerns the energy flux balance equation (40). The third term can be simplified as follows:

$$
\frac{\partial}{\partial c}\left(\frac{1}{2} m c^{2} c\right)=m c \frac{\partial c}{\partial c} c+\frac{1}{2} m c^{2} \frac{\partial c}{\partial c}=m c c+\frac{1}{2} m c^{2} \boldsymbol{I},
$$

where $I$ is the unity tensor. Assuming again as in (46) that the temperature tensor is isotropic, and hence that

$$
\langle c c\rangle \approx \frac{\left\langle c^{2}\right\rangle}{3} I
$$

and after some algebra, the energy flux equation can be written as

$$
\frac{\partial}{\partial t}(n \boldsymbol{\xi})+\nabla \cdot\left(n\left\langle\frac{1}{2} m c^{2} c c\right\rangle\right)-\frac{e}{m} \boldsymbol{E}\left(\frac{5}{3} n \varepsilon\right)=-n \widetilde{v}_{m} \boldsymbol{\xi}
$$

The second term in the energy flux balance equation (49) or (40) is the divergence of the fourth power of the velocity averaged over the velocity distribution, $\left\langle c^{2} c c\right\rangle$, while all other terms in the equation contain only the third power of velocity. Therefore, this term, which we will call the quartic tensor, requires either the next equation in the sequence of moment equations or a closure approximation. This closure assumption must be physically transparent and consistent with the general structure of the equations. One way is to approximate the relevant term by a product of lower moments as

$$
\left\langle c^{2} c c\right\rangle \approx \beta\left\langle c^{2}\right\rangle\langle c c\rangle \approx \beta \frac{4}{3 m^{2}} \varepsilon^{2} I,
$$

where we used (48) for the second equality. $\beta$ is a parametrization factor, generically close to unity, when the higher order correlation term $\left\langle c^{2} c c\right\rangle-\left\langle c^{2}\right\rangle\langle c c\rangle$ can be neglected.

With these closure assumptions the system of fluid equations (37)-(40) for the electrons becomes

$\frac{\partial n}{\partial t}+\nabla \cdot n \boldsymbol{v}=-n\left(\widetilde{v}_{\mathrm{A}}-\widetilde{v}_{\mathrm{I}}\right)$, 


$$
\begin{aligned}
& \frac{\partial}{\partial t}(n \boldsymbol{v})+\frac{2}{3 m} \nabla(n \varepsilon)-n \frac{e}{m} \boldsymbol{E}=-n \boldsymbol{v}\left(\widetilde{v}_{m}+\widetilde{v}_{\mathrm{I}}+\frac{2}{3 m} \varepsilon \widetilde{v}_{\mathrm{A}}^{\prime}\right) \\
& \frac{\partial}{\partial t}(n \varepsilon)+\nabla \cdot(n \boldsymbol{\xi})-e \boldsymbol{E} \cdot(n \boldsymbol{v}) \\
& =-n \widetilde{v}_{e}\left[\left(\varepsilon-\frac{3}{2} k T_{0}\right)+\Omega\right]-n \varepsilon \widetilde{v}_{\mathrm{A}} \\
& \frac{\partial}{\partial t}(n \boldsymbol{\xi})+\nabla\left(\beta \frac{2 n}{3 m} \varepsilon^{2}\right)-\frac{5}{3} n \varepsilon e \boldsymbol{E}=-\widetilde{v}_{m}(n \boldsymbol{\xi})
\end{aligned}
$$

The parameter $\beta$ that appears in the energy flux equation is a quantity close to unity, but can be used to fit the neglected higher order correlations. Variations of this parameter will be discussed in our second paper [43].

The system of equations (51)-(54) has the following properties. (i) If the parameter $\beta$ is specified and if the collision terms are given then the system of equations containing no further unknowns can be numerically solved for the density, average velocity, average energy and energy flux. (ii) The equations contain mean-energy collisional rates that should be carefully derived and implemented as elaborated in the next section. (iii) Attachment enters the momentum and energy balance equation in terms of the derivative of the attachment collision frequency while for ionization only the ionization collision frequency is present. (iv) The above equations are set for a single component gas; the generalization to gas mixtures proceeds through the generalization of the collision term to a sum of terms appropriately weighted according to the mole fractions of the respective species.

\section{Transport and reaction data}

\subsection{Evaluation of the transport data: Boltzmann equation analysis}

Here we discuss how to evaluate and implement electron transport properties in both first- and high-order fluid models. In plasma modelling, transport coefficients of electrons and/or ions may be obtained either from swarm experiments, from solutions of the Boltzmann equation or from Monte Carlo simulations. We note that swarm transport data are usually given in the literature in the form of tables as a function of reduced electric field, $E / n_{0}[36,44]$. In fluid plasma modelling, however, several important issues have to be considered before directly implementing data from the literature.

First, transport coefficients are defined far from boundaries, sources and sinks of charged particles where the so-called hydrodynamic conditions prevail $[36,37,39$, $40,62,63,69]$. Under hydrodynamic conditions, the spacetime dependence of the distribution function is expressible in terms of linear functionals of $n(\boldsymbol{r}, t)$. A sufficient functional relationship between the distribution function $f(\boldsymbol{r}, \boldsymbol{c}, t)$ and $n(r, t)$ in the case of weak gradients is the well-known expansion

$$
f(\boldsymbol{r}, \boldsymbol{c}, t)=\sum_{s=0}^{\infty} f^{(s)}(\boldsymbol{c}) \odot(-\nabla)^{s} n(\boldsymbol{r}, t),
$$

where $f^{(s)}(\boldsymbol{c})$ are tensors of rank $s$ and $\odot$ denotes an $s$-fold scalar product. Direct application of transport properties measured/calculated in different experimental arrangements, where often non-hydrodynamic conditions are present explicitly or implicitly, is problematic and should be avoided. Typical examples of swarm data obtained under nonhydrodynamic conditions are those measured/calculated under the steady-state Townsend (SST) conditions [64, 65]. Before direct application of SST data one must perform careful swarm analysis and convert the SST data into the hydrodynamic transport coefficients. Details of this procedure are presented in [66].

Second, care must be taken when non-conservative collisions are significant. In the presence of non-conservative collisions there are two sets of transport coefficients, the bulk and the flux $[39,67]$. Assuming the functional relationship (55) the flux $\Gamma$ and source term $C_{1}$ (usually denoted by $S(r, t)$ in previous swarm-oriented studies $[36,37,62,63])$ appearing in the equation of continuity (7) can be expanded

$\boldsymbol{\Gamma}(\boldsymbol{r}, t)=n \boldsymbol{W}^{(\star)}-\boldsymbol{D}^{(\star)} \cdot \nabla n$,

$S(\boldsymbol{r}, t)=S^{(0)} n(\boldsymbol{r}, t)-\boldsymbol{S}^{(1)} \cdot \nabla n(\boldsymbol{r}, t)+\boldsymbol{S}^{(2)}: \nabla \nabla n(\boldsymbol{r}, t)$,

where $\boldsymbol{W}^{(\star)}$ and $\boldsymbol{D}^{(\star)}$ define, respectively, the flux drift velocity and flux diffusion tensor while $\boldsymbol{S}^{(k)}$ are expansion coefficients of the source term. Substitution of expansions (56) and (57) into the continuity equation (7) yields the diffusion equation

$$
\frac{\partial n}{\partial t}+\boldsymbol{W} \cdot n-\boldsymbol{D}: \nabla \nabla n=-R_{a} n
$$

which defines the bulk transport coefficients

$$
\begin{gathered}
R_{a}=-S^{(0)}, \\
\boldsymbol{W}=\boldsymbol{W}^{(\star)}+\boldsymbol{S}^{(1)}, \\
\boldsymbol{D}=\boldsymbol{D}^{(\star)}-\boldsymbol{S}^{(2)},
\end{gathered}
$$

where $R_{a}$ is the loss rate, $W$ is the bulk drift velocity and $D$ is the bulk diffusion tensor.

The basic difference between the bulk and flux transport coefficients should now be apparent. The bulk drift velocity is the displacement of the mean position of the electron swarm and it characterizes the motion of the total ensemble of electrons. The presence of the electric field results in a spatial variation in the energy throughout the swarm. Under such conditions, the presence of non-conservative collisions (ionization/attachment) may lead to a change in the position of the centre-of-mass of the swarm. This effect on the bulk drift velocity is denoted by $\boldsymbol{S}^{(1)}$. On the other hand, the flux drift velocity $\boldsymbol{W}^{(\star)}$ represents the rate of change of the position of the centre-of-mass due to the electric field only and can be interpreted as the mean velocity of the electrons. Likewise, the flux diffusion tensor $\boldsymbol{D}^{(\star)}$ represents the rate of spreading of the swarm due to the electric field $\boldsymbol{E}$ and gradients in density $\nabla n$. The presence of non-conservative collisions may result in the variation of $\nabla n$ throughout the swarm and a subsequent variation in the rate of change of the mean squared width of the swarm. Such effects are expressed by the second 
rank tensor $\boldsymbol{S}^{(2)}$. The most appropriate procedure in plasma modelling would be to use the experimental swarm data (e.g. bulk values) for the analysis of the validity of the cross-section and then to calculate the flux quantities, which are necessary as input data in fluid modelling. More about duality of the hydrodynamic transport coefficients and their implementation in fluid modelling can be found in [29, 36, 37, 44, 62, 63].

Third, while the first-order fluid model requires electron transport data as a function of local reduced electric field, what really appears in high-order fluid models are mean-energydependent collisional rates. Since momentum transfer theory is used to determine the collision terms in the fluid equations, the most appropriate procedure would be to systematically reduce these fluid equations down to the swarm limit assuming the hydrodynamic regime. From this set of equations, one can then find relationships between collisional transfer rates and the mean energy, in a self-consistent manner. This method was employed in previous works of Robson and co-workers (see for example [44] and references therein), but we apply here a slightly different approach. Instead of using the socalled generalized Einstein relation to determine the mean energy from the transverse diffusion coefficient [44,47], the mean energy is directly calculated from the multi-term solution of Boltzmann's equation. The correspondence between the mean energy and $E / n_{0}$ is then used to find the correspondence between the mean energy and other relevant transport data. The momentum transfer collision rate is obtained from

$$
v_{m}=\frac{e}{m \mu(\varepsilon)},
$$

where $\mu(\varepsilon)$ is the electron mobility which is here a function of the mean energy. The energy-transfer collision frequency is calculated from equation (43). Thus, our procedure of determining the electron transport data as an input for highorder fluid models is entirely consistent with the work of Boeuf and Pitchford [68].

The electron transport data employed in this work are calculated using a multi-term theory for solving the Boltzmann equation. The methods and techniques are by now standard and the reader is referred to our previous works [36, 37, 62]. Among many important aspects, we highlight the following important steps:

- No assumptions on symmetries in velocity space are made, and the directional dependence of the phase-space distribution function in velocity space is represented in terms of a spherical harmonic expansion:

$$
f(\boldsymbol{r}, \boldsymbol{c}, t)=\sum_{l=0}^{\infty} \sum_{m=-l}^{l} f(\boldsymbol{r}, c, t) Y_{m}^{[l]}(\hat{\boldsymbol{c}}),
$$

where $Y_{m}^{[l]}(\hat{\boldsymbol{c}})$ are spherical harmonics and $\hat{\boldsymbol{c}}$ represents the angles of $c$. In contrast to the classical two-term theory (where the sum over $l$ is performed only up to $l=1$ ), the number of spherical harmonics is not restricted, and our method therefore is a truly multi-term approach. The differences between the two-term approximation and our full multi-term approach for electron transport in nitrogen are illustrated in section 3. The inadequacies of a Legendre polynomial expansion (when density gradients are not parallel to the field) are highlighted in our previous publications [36,37] and avoided in this work.

- As discussed above, under hydrodynamic conditions a sufficient representation of the space dependence is an expansion in terms of powers of the density gradient operator.

- The velocity (energy) dependence of the phase-space distribution function is represented by an expansion about a Maxwellian at an arbitrary temperature in terms of Sonine polynomials.

Using the appropriate orthogonality relations for the spherical harmonics and for the modified Sonine polynomials, the Boltzmann equation is converted into a hierarchy of coupled equations for the moments of the distribution function. These equations are solved numerically and all transport and rate coefficients are expressed in terms of moments of the distribution function $[36,37]$.

\subsection{Cross-sections and transport data}

In this section, transport and reaction coefficients for electrons in $\mathrm{N}_{2}$ at a temperature of $298 \mathrm{~K}$ are calculated as an input for first- and high-order fluid models. The first-order model is based on the local field approximation; it requires mobility, diffusion coefficient and ionization rate as a function of the reduced electric field $E / n_{0}$ (where $n_{0}$ is the gas number density). Compared with our previous work [62], we extend the electric field range up to $3000 \mathrm{Td}$ $\left(1 \mathrm{Td}=10^{-21} \mathrm{~V} \mathrm{~m}^{2}\right)$. The high-order fluid model requires average collision frequencies for momentum and energy transfer in elastic and inelastic collisions, and rate coefficients for all collision processes as a function of the mean electron energy.

We use the cross-sections for electron scattering in $\mathrm{N}_{2}$ provided by Stojanović and Petrović [70]. For elastic collision processes we use the original Boltzmann collision operator [71] while for inelastic processes we employ the generalization of Wang-Chang et al [72]. The ionization collision operator is detailed in [40]. We assume that the ratio between the energy of the scattered electron and the total available energy in an ionizing collision is equally distributed between 0 and 1; the same holds then, of course, for the ejected electron. We remark that at the high electron energies in the streamer tip, the assumptions on the energy division can considerably influence transport profiles. Furthermore, scattering is assumed to be isotropic. This can be problematic for high values of $E / n_{0}$ (generally for $E / n_{0} \geqslant 1000 \mathrm{Td}$ for electrons in $\mathrm{N}_{2}$ ) when electrons scatter predominantly in the forward direction [24,75]. However, the errors in the calculated transport coefficients and rate coefficients are acceptable in fluid modelling for streamers in the range of the reduced electric fields $E / n_{0}$ considered in this work after appropriate renormalization of cross-sections for the scattering angle distribution [24].

We present both bulk and flux coefficients obtained by our multi-term solution of the Boltzmann equation, 


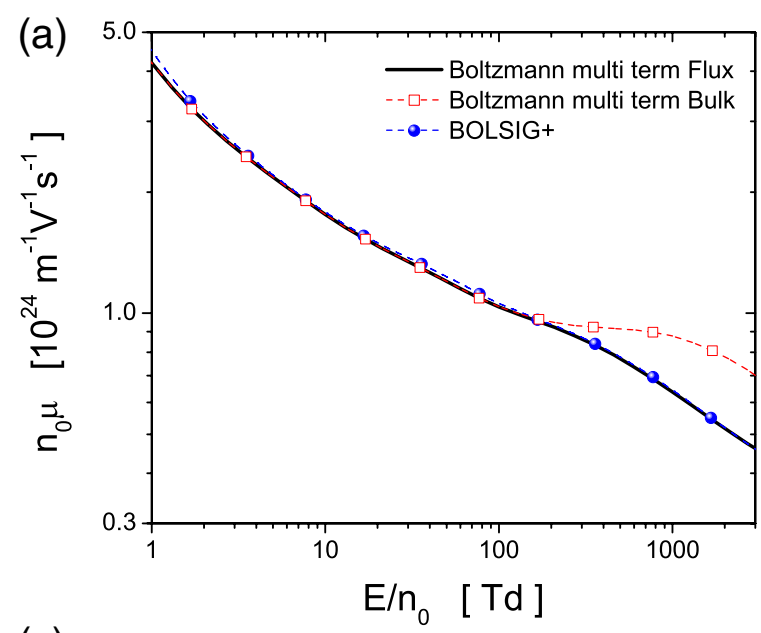

(c)

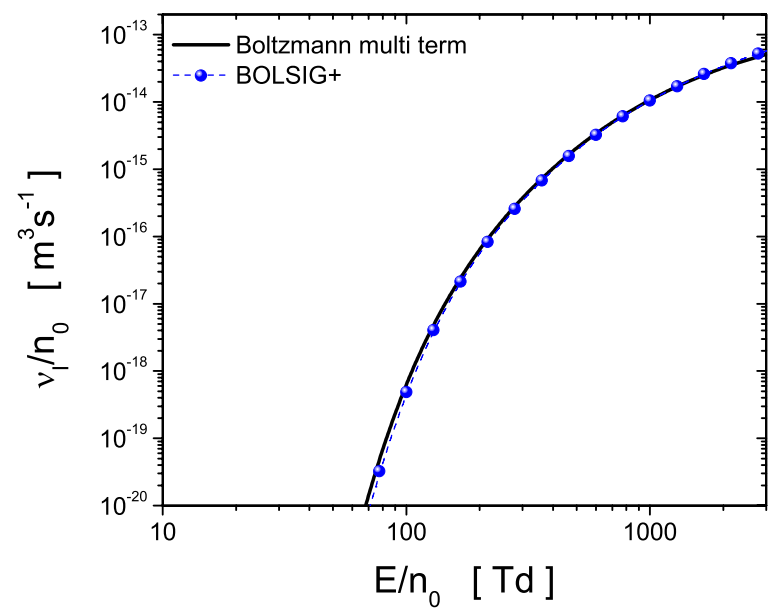

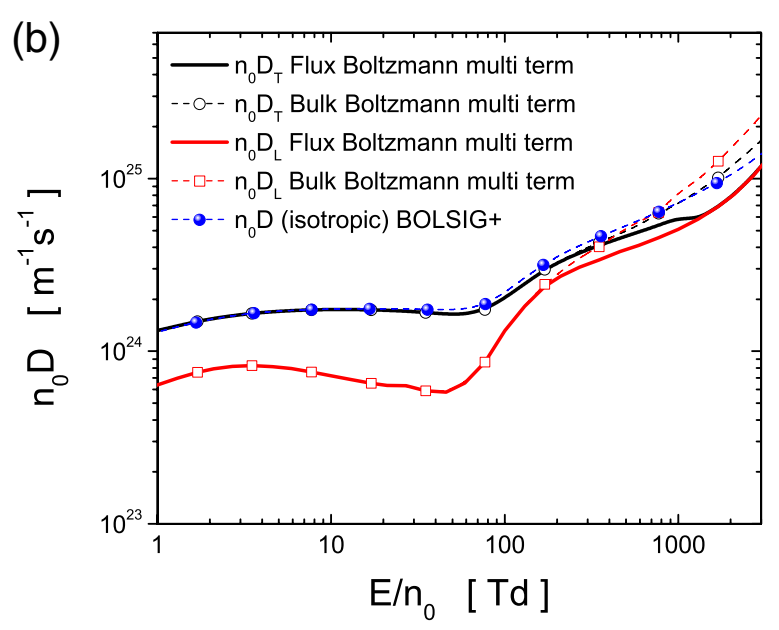

(d)

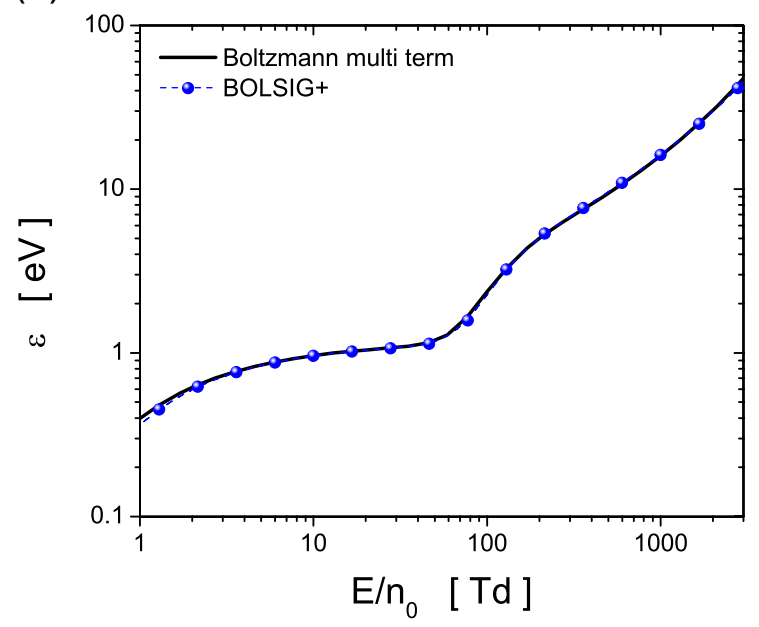

Figure 1. (a) Mobility, (b) longitudinal and transverse diffusion coefficient, and (c) ionization rate of electrons in $\mathrm{N}_{2}$ as a function of the reduced electric field $E / n_{0}$, as an input for the first-order model. Shown are flux and bulk data obtained by our multi-term solution of the Boltzmann equation, and flux data obtained by the BOLSIG+ code. BOLSIG+ provides only an isotropic diffusion coefficient for $(b)$.

$(d)$ shows the variation of mean energy with $E / n_{0}$.

and we compare them with the coefficients obtained by the public available Boltzmann solver BOLSIG+ derived from the same cross-sections. BOLSIG+ is based on the two-term approximation [38] and provides exclusively flux transport data.

\subsection{Input data for the first order model}

Figure 1( $a$ ) shows the electron mobility (multiplied by the gas number density) as a function of the reduced electric field $E / n_{0}$. We observe that bulk and flux quantities start to differ visibly above a reduced field of approximately $150 \mathrm{Td}$, this means that the ionization processes start to be significant at this value of the field. As $E / n_{0}$ increases further, the effect becomes more pronounced, until the bulk mobility exceeds the flux mobility by approximately $30 \%$ at $3000 \mathrm{Td}$. The difference between bulk and flux mobility is the consequence of the spatial variation of the average electron energy within an electron swarm $[32,37,40]$. If the ionization rate is an increasing function of electron energy (as is the case for the parameters considered here), electrons are preferentially created in regions of higher energy resulting in a shift in the centre-of-mass position as well as in a modification of the spread about the centre-of-mass. In nitrogen up to $3000 \mathrm{Td}$, the electrons are preferentially created at the leading edge of an electron swarm and hence the bulk mobility is larger than the flux mobility.

Figure 1(a) shows as well that the flux data obtained by our multi-term solution of Boltzmann's equation and by the BOLSIG+ code agree well. Only for $E / n_{0}$ below about $3 \mathrm{Td}$ is the BOLSIG+ mobility higher than our flux mobility. As we have successfully compared our multi-term results with Monte Carlo results that include the thermal energy of the background molecules as well, the results shown in figure 1(a) suggest that the BOLSIG+ code should be carefully tested in the limit of thermal energies.

Figure 1(b) shows the diffusion coefficients (multiplied by the gas number density) as a function of the reduced field $E / n_{0}$. The bulk and flux values of the longitudinal and transverse diffusion coefficients are compared with the isotropic diffusion coefficient calculated by the BOLSIG+ code. As for the mobility, flux and bulk data start to deviate for $E / n_{0}$ above approximately $150 \mathrm{Td}$, indicating again the onset of ionization effects. The diffusion coefficients are more sensitive to the 

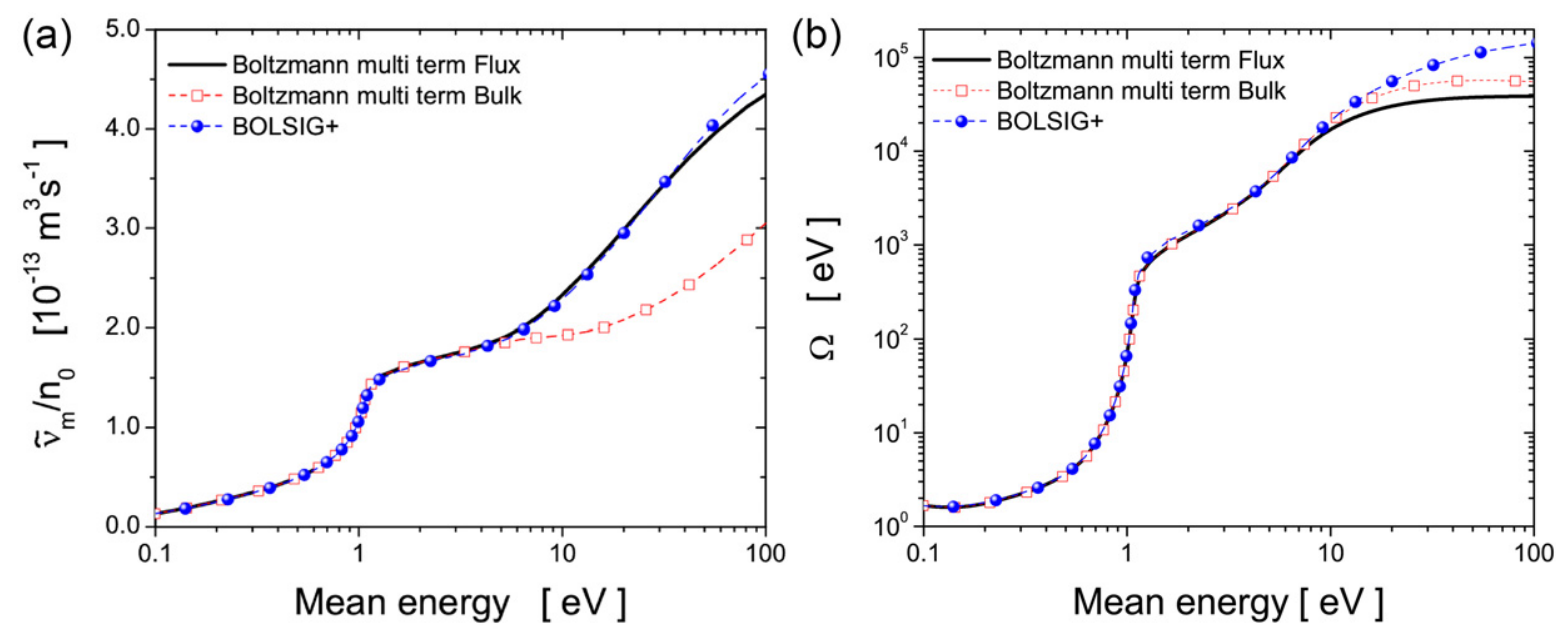

Figure 2. Density normalized average collision frequency for momentum transfer in elastic collisions $\widetilde{v}_{m} / n_{0}(a)$ and the average energy lost in one energy relaxation time $\widetilde{v}_{e}^{-1}$, through non-elastic processes $\Omega(b)$ as a function of the mean energy of electrons in $\mathrm{N}_{2}$, as an input for the high-order model. The three curves in each panel show the flux and bulk data obtained by our multi-term solution for the Boltzmann equation and the flux data obtained by the BOLSIG+ code.

ionization processes than the mobility; the differences between bulk and flux data can reach almost $50 \%$ for $E / n_{0}$ approaching $3000 \mathrm{Td}$. For a more thorough analysis of the explicit influence of ionization processes on the diffusion coefficients one must consider the second-order variations in the average energy along the swarm. This is beyond the scope of this work and we defer this to a future study.

Figure $1(b)$ clearly shows the anisotropy of the diffusion tensor, i.e. $D_{\mathrm{L}} \neq D_{\mathrm{T}}$; for the range of $E / n_{0}$ considered here, the transverse diffusion coefficient is always larger than the longitudinal diffusion coefficient. This is due to the spatial variation of the average energy within the swarm and to the energy dependence of the collision frequency. The theory of anisotropic diffusion in an electric field is now textbook material (see for example [73]) and rather than a detailed discussion of the origin of this phenomenon, we prefer to highlight the implementation of the diffusion coefficients in fluid models of streamer discharges. For $E / n_{0}$ below approximately $30 \mathrm{Td}$, our results for the flux component of the transverse diffusion coefficient and those obtained by the BOLSIG+ code agree very well. For $E / n_{0}$ above $30 \mathrm{Td}$, however, BOLSIG+ with its two-term approximation is clearly above our multi-term solution. Here one should bear in mind that BOLSIG+ treats the diffusion processes under the spatially homogeneous conditions and hence the transverse diffusion coefficient obtained under the spatially inhomogeneous conditions should be used for comparisons. On the other hand, our one-dimensional fluid model requires the longitudinal diffusion coefficient as an input as we consider the spatial variations of the electron density and average electron energy only along the field direction. Therefore, particular care needs to be taken with implementation of the diffusion coefficients in fluid models of streamer discharges.

Figure 1(c) shows that the ionization rate (divided by the gas number density) differs between the two-term and the multi-term calculations by up to $30 \%$. It is interesting to note that the two-term approximation is less accurate in the energy region dominated by the vibrational excitation of $\mathrm{N}_{2}$ and for energies well above the ionization threshold. Surprisingly, for the electric field range between approximately 200 and $600 \mathrm{Td}$ the two-term approximation increases in accuracy. In this energy region, the cross-sections for inelastic processes are much smaller than for elastic collisions. Similar but not identical observations have been made by Phelps and Pitchford [75].

Figure $1(d)$ shows the variation of the mean energy with $E / n_{0}$. The properties of the cross-sections are reflected in the profile of the mean energy. The initial slow rise indicates the influence of low-threshold rotational and vibrational processes. The sharp rise for mean energies starting approximately from $50 \mathrm{Td}$ indicates the 'turning off' of the vibrational processes. Our results and those obtained by the BOLSIG+ code agree very well. The mean energy is determined from the balance between the gain from the field and the loss by collisions with the molecule. This quantity is directly used in high-order fluid modelling of streamer discharges. Although it does not appear in the first-order fluid models, unlike other transport coefficients such as the drift velocity (mobility), diffusion coefficients and ionization rate, we often find that a knowledge of the mean energy is necessary to explain certain phenomena.

\subsection{Input data for the high-order model}

The density normalized average collision frequencies for momentum transfer in elastic collisions and the average energy loss in one energy relaxation time $\widetilde{v}_{e}^{-1}$, through non-elastic processes as a function of mean electron energy are shown in figure 2. Bulk and flux frequencies obtained with the multi-term approach are compared with flux values calculated with BOLSIG+. The flux values of the two approaches for $\widetilde{v}_{m} / n_{0}$ agree well. The differences between bulk and flux values of $\widetilde{v}_{m} / n_{0}$ are a direct consequence of the differences between bulk and flux mobilities (see figure 1(a)). Similar arguments can be used to explain the difference between bulk and flux components of $\Omega$. This quantity should be viewed 

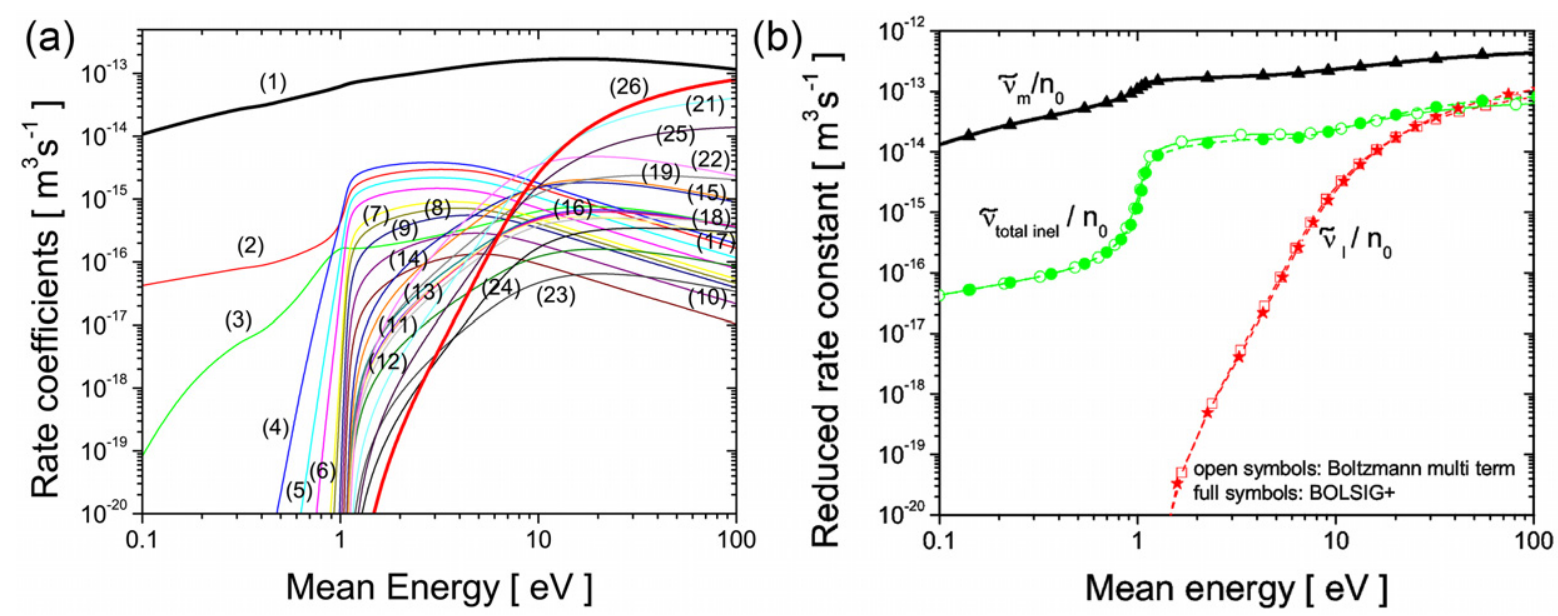

Figure 3. Rate coefficients in $\mathrm{N}_{2}$ as a function of the electron energy, calculated with the multi-term solution of the Boltzmann equations. (a) Rates for the cross-sections listed in [70,75]: momentum transfer in elastic collisions (1), rotational excitation (2), vibrational excitations (3-11), electronic excitation $A^{3} \Sigma_{\mathrm{u}}^{+} v=(0-4)(12), A^{3} \Sigma_{u}^{+} v=(5-9)(13), B^{3} \Pi(14), W^{3} \Delta(15), A^{3} \Sigma_{u}^{+} v>10(16), B^{\prime} \Sigma_{u}^{-}$ (17), $a^{\prime} \Sigma_{\mathrm{u}}^{-}$(18), $a^{1} \Pi_{\mathrm{g}}(19), w^{1} \delta_{\mathrm{u}}(20), C^{3} \Pi_{\mathrm{u}}(22), E^{3} \Sigma_{\mathrm{g}}^{+}(23), a^{\prime \prime}{ }^{1} \Sigma_{\mathrm{g}}^{+}$(24), sum of singlets (25), dissociation [74] (21), ionization (26); and (b) density normalized momentum transfer rate, density normalized total inelastic rate and ionization rate.

as a 'measure' of energy transfer in inelastic collisions in one energy relaxation time $\widetilde{v}_{e}^{-1}$. It is directly proportional to mobility and hence its bulk dominates the corresponding flux component for higher mean electron energies. Differences between flux values of the two approaches for $\Omega$ follow directly from internal errors of the two-term approximation used to solve Boltzmann's equation in the BOLSIG+ code.

Figure 3(a) shows the rate coefficients as a function of the mean electron energy for all collision processes. Figure 3(b) compares the density normalized average collision frequency for momentum transfer in elastic collisions, the density normalized total inelastic rate (sum of all rates for inelastic processes without ionization) and the ionization rate. All results are obtained with the solution of Boltzmann's equation. On figure $3(b)$ the results obtained by the BOLSIG+ code are also included. The ionization rate is significant for relatively high mean energies (i.e. high $E / n_{0}$ ) and is essential for modelling of streamers. Excepting rates for dissociation (21), electronic excitation (25) and ionization (26), the following feature is observed in the mean-energy profiles of the rate coefficients: at some point their increase with the mean energy slows down until a maximum is reached and then they start to decrease. Under conditions considered in this work, the ionization rate monotonically increases with the mean energy and in the limit of $100 \mathrm{eV}$ it approaches the rate for momentum transfer in elastic collisions. In the low-energy range, however, rotational and vibrational excitations have the most important role. In particular, for electron energies less than approximately $0.1 \mathrm{eV}$, the processes of rotational excitation control the energy transfer in the system and should be carefully considered. In conclusion, collisional rates for inelastic processes enter the energy balance equation describing the change in the average energy of electrons. The knowledge of the rate coefficients is of key importance to calculate the kinetics of various plasma chemical processes and densities of excited species and thus used in much broader context of plasma modelling.

\section{First-order streamer model with different transport data}

Here we present simulations of planar negative ionization fronts in $\mathrm{N}_{2}$ using the first-order fluid model, while simulation results with the high-order fluid model are presented in our second paper [43].

\subsection{Numerical methods, initial and boundary conditions}

In this subsection we briefly describe the numerical method, and the initial and boundary conditions used to solve equations (25)-(28) in one spatial dimension. The calculations are carried out in $\mathrm{N}_{2}$ at atmospheric pressure and at the ambient temperature of $298 \mathrm{~K}$. The 1D simulations are started with the same initial Gaussian-type distribution for electrons and ions

$$
\left.n(x)\right|_{t=0}=n_{i} \exp \left[-\frac{\left(x-x_{0}\right)^{2}}{\sigma^{2}}\right],
$$

in a gap parametrized with the coordinate $x \in[0, L]$, with $L=1.2 \mathrm{~mm}$. We have chosen $n_{i}=2 \times 10^{18} \mathrm{~m}^{-3}$, $x_{0}=8 \times 10^{-4} \mathrm{~m}$ and $\sigma=2.9 \times 10^{-5} \mathrm{~m}$. The externally applied electric field is positive in the $x$ direction, therefore electrons drift to the left. The field is fixed in the non-ionized region at the left boundary $x=0$, providing a fixed electric field for the negative streamer ionization front to penetrate. In this work we consider reduced electric fields of 350, 460, 590, 770 and $1000 \mathrm{Td}$.

To investigate the sensitivity of streamer properties to the definition and accuracy of the transport data, we employ three different sets of data for $\mu, D$ and $\nu_{\mathrm{I}}$ : bulk and flux data obtained by our Boltzmann equation analysis and the flux data obtained by the BOLSIG+ code.

The finite volume method is used to spatially discretize the system (25)-(28) on a uniform grid with 1000 points. More details will be outlined in our second paper [43], where 
numerical methods for solving the high-order fluid equations are presented in a comprehensive way. To approximate the spatial derivatives in (25) we use the second-order central difference discretization while the time derivatives are approximated with the Runge-Kutta 4 method [76]. The continuity equation for the electron density has a second-order spatial derivative, and therefore it requires two boundary conditions. For $x=0$ we use a homogeneous Neumann boundary condition $\left(\partial_{x} n=0\right)$, so that electrons that arrive at this boundary may flow out of the system. For $x=L$ we employ a homogeneous Dirichlet boundary condition $(n=0)$ to ensure that there is no outflow of electrons from the system. In any case, it should be noted that the electrons are well separated from the boundaries, therefore the actual boundary conditions do not matter. In equation (26) the time derivative is approximated with the Runge-Kutta 4 method using the same time step as in equation (25). In the 1D case, equation (28) has the form

$$
\partial_{x} E=\frac{e}{\epsilon_{0}}\left(n-n_{\text {ion }}\right),
$$

from which we can determine the electric field $E$ by integrating over $x$ and using the fixed value of $E$ at $x=0$.

\subsection{Overview of simulation results with different transport data}

Figure 4 displays the spatio-temporal evolution of electron and ion densities and of the electric field when the reduced electric field ahead of the front is fixed to $590 \mathrm{Td}$ (or equivalently to $145 \mathrm{kV} \mathrm{cm}^{-1}$ in $\mathrm{N}_{2}$ at atmospheric pressure and temperature of $298 \mathrm{~K}$ ). Calculations are performed for three different sets of transport data as indicated in the figures. We start with a Gaussian density distribution as described above. Although the transition from avalanche to streamer has been discussed many times within the past 80 years [32,77-79], the characteristics and main physical processes are discussed here to investigate the sensitivity to different transport data.

In the early stage of evolution, we see that both the electron and the ion densities grow due to electron impact ionization. If this were the only mechanism, the electric field would remain unchanged and the ionization would continue indefinitely. However, the electrons drift in the direction opposite to the electric field while the positive ions would slowly drift in the opposite direction; as their mobility is so much smaller, this motion is actually neglected here and in most other streamer studies. As a consequence, the charge separation starts to distort the initially homogeneous electric field. Now figure 4 shows that the ionization profiles at time $0.06 \mathrm{~ns}$ obtained with the bulk transport data are somewhat wider while their height is less than with our flux and BOLSIG+ data. This follows from the fact that the bulk mobility and diffusion constant are higher than the corresponding flux data and hence the centreof-mass moves faster and the electron package spreads faster. As the ionization rate is the same in both cases, the height of the profiles obtained with the bulk data must be less than with the flux and BOLSIG+ data. As the evolution continues, the electric field in the ionized region gets completely screened, and further ionization processes cannot occur in this region anymore. The transition from avalanche to streamer is then completed. We mention in passing that the complete screening of the interior field is due to the 1D set-up and to the fact that the field ahead of the front does not change in time.

\subsection{Front velocities}

When the streamers have approached an approximately uniformly translating state, we see that the streamers obtained with bulk data propagate faster than those obtained with flux and BOLSIG+ data, in agreement with earlier studies $[23,25,32]$. In section 3.1 , it was already discussed that in general flux transport data should be used in fluid equations derived systematically from the Boltzmann equation; however, in the particular case of streamer ionization fronts with their pulled dynamics simulated with the half-phenomenological classical streamer model, the bulk coefficients approximate the front velocity better, with the drawback that the ionization level behind the front is very small $[23,25,32]$.

In figure 5 we display velocities of planar fronts as a function of $E / n_{0}$. In order to calculate the streamer velocity we have followed the evolution of a certain level $\left(2 n_{i}\right)$ of the electron density at the streamer front. We compare the velocities obtained with different input data. The flux drift velocity as a function of $E / n_{0}$ is also shown. First, we see that the planar fronts move much faster than the electrons. This follows from the fact that the velocity of a planar front is the sum of the drift in the electric field at the front edge plus a term accounting for diffusion, for creation of additional electrons due to impact ionization and for the electron density profile [42]. The difference between front velocity and electron drift velocity increases with $E / n_{0}$, up to more than a factor of 2 for the highest field displayed here.

We remark that according to analytical theory [42,80], the front velocities in planar configurations (where the field does not decay ahead of the front) depend for a long time on initial conditions, and if the initial condition decays less than $\mathrm{e}^{-\Lambda^{*}|x|}, \Lambda^{*}=\sqrt{v_{\mathrm{I}} / D}$, it will determine the front velocity for arbitrarily long times. For this reason, here we do not compare numerical with analytical results [42].

We finally note that the front velocities calculated with bulk transport data are up to $30 \%$ higher than with flux data. This illustrates the sensitivity of the model to the input data. On the other hand, the velocities differ much less between our flux data and those obtained with BOLSIG+. To explore this issue in more detail, additional tests are required, particularly for atomic and molecular systems with large anisotropy of the velocity distribution function in velocity space.

\subsection{Ionization levels behind the front}

We now explore how the ionization degree behind the front depends on the transport data, and we compare with the analytical approximation

$$
n_{e, \text { back }} \leqslant \frac{\epsilon_{0}}{e} \int_{0}^{E_{\max }} \frac{v_{\mathrm{I}}(E)}{E \mu(E)} \mathrm{d} E .
$$

This approximation becomes an identity, if diffusion can be neglected [42]; and it is an upper bound, if diffusion 

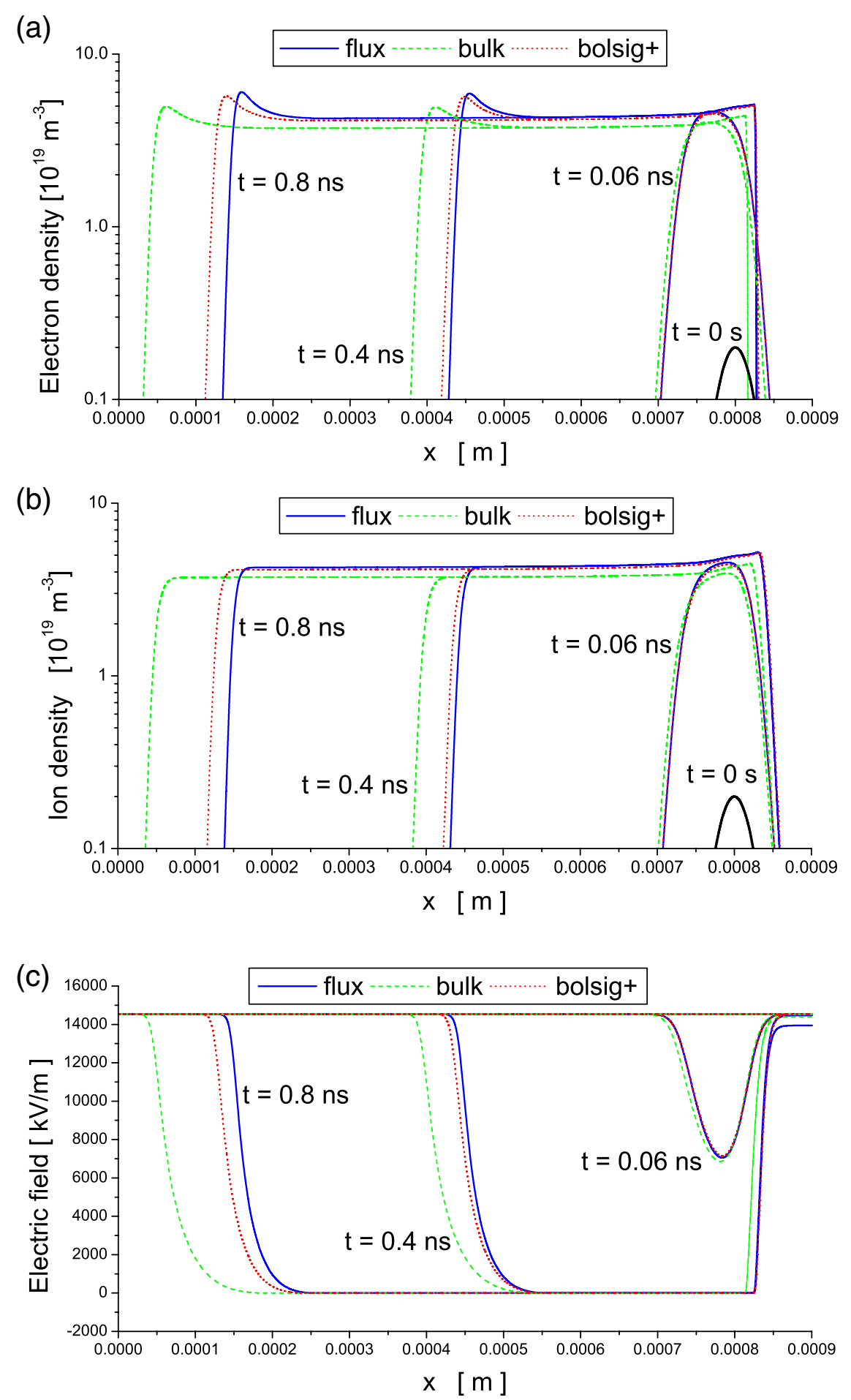

Figure 4. Evolution of $(a)$ the electron density, $(b)$ the ion density and $(c)$ the electric field in a negative planar ionization front in $\mathrm{N}_{2}$ with a reduced electric field of $590 \mathrm{Td}$ ahead of the front. Shown are the spatial profiles obtained with three different sets of input data as indicated in the graph.

is taken into account [32]. This analytical result has been derived for planar fronts, and it is independent of the front velocity.

In figure 6 we compare the ionization levels behind the fronts calculated with the three different sets of transport data and with the analytical upper bound, using our flux data. We see that the approximation (66) indeed serves as such a bound, but is furthermore also a very good approximation of the numerical results, when using the same transport data. The errors of the two-term approximation are negligible indicating a weak sensitivity of the ionization level to the isotropy of the distribution function in velocity space that is assumed in BOLSIG+. The ionization level with bulk data is considerably lower. Generally, it is evident that the ionization level is much less sensitive to the type of transport data than the front velocity. Similar observations have been made in [32]. 


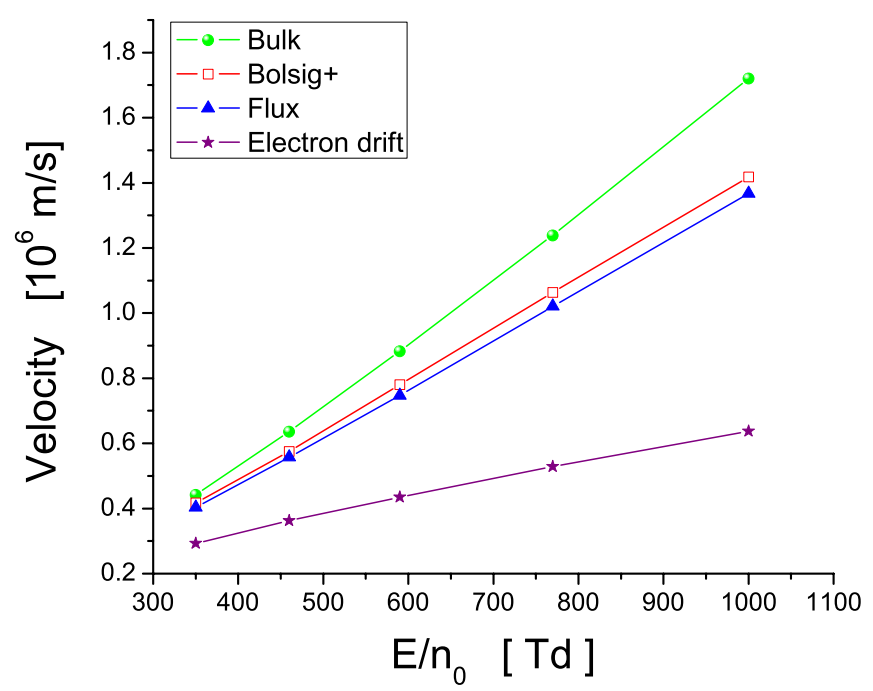

Figure 5. Velocities of planar fronts obtained by three different sets of input data as a function of the reduced electric field. The flux drift velocity of electrons is also included.

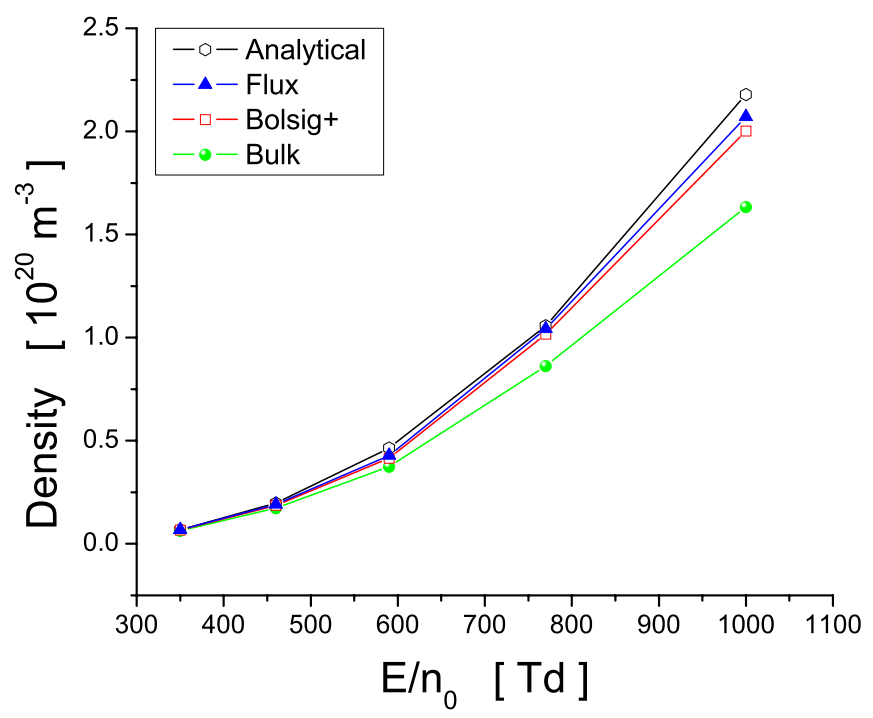

Figure 6. Ionization levels behind planar fronts as a function of the reduced electric field. Results are shown with three different sets of input data and with the analytical approximation (66) calculated with flux data.

\section{Conclusion}

In this paper, we have derived a high-order fluid model for streamer discharges. Our goal has been to develop a comprehensive theory at a level of sophistication appropriate for the electron energy distributions very far from equilibrium and for the steep electron density gradients that characterize a streamer front. The first steps are reported in this paper, which deals with theoretical foundations and phenomenology. After examining the application of the Boltzmann equation, we have proceeded to the evaluation of the velocity moments of Boltzmann's equation. To make contact with previous works and to place our theory in a much broader context, we have demonstrated how to derive the classical drift-diffusion approximation often used in the plasma modelling community to model streamer discharges. Then we have introduced our more sophisticated high-order fluid approach, proceeding directly from Boltzmann's equation and systematically discussing the critical assumptions required to close the system of equations and to evaluate the collision terms involved. Momentum transfer theory has been used to approximate collision terms in the high-order fluid model while highorder tensors appearing in the energy flux equation have been specified in terms of previous moments. In contrast to previous works, it has been emphasized that the energy flux equation plays a pivotal role for the correct description of streamer dynamics. The fluid equations obtained as velocity moments of the Boltzmann equation have been closed in the local mean energy approximation and coupled to the Poisson equation to calculate the modification of the electric field by space charges. The numerical solutions of the high-order fluid model for planar fronts and their discussion are deferred to the following paper.

The second important aspect of this paper concerns the application of transport data in fluid models of streamer discharges. In order to illustrate this issue, we have used the first-order fluid model to investigate the temporal evolution of negative planar fronts in pure nitrogen. We have focused on the way in which the inherent streamer properties such as the velocity of a streamer or the ionization level behind the front are influenced by different transport data employed as an input in fluid equations. Our primary goal was to show which aspects of kinetic theory developed for swarm physics and particularly which segments of data would be important for further improvement of streamer models. It was shown and illustrated that the direct application of transport data from the literature without knowledge of origin and nature of the data is problematic and can often lead to significant errors in the profiles of various streamer properties. In this respect, the origin and nature of transport data must be known and, if appropriate, suitably modified before implementation in the fluid models. This is particularly important for collisional transfer rates required as input in the high-order fluid model. We have also discussed the validity of transport data obtained by a two-term theory for solving the Boltzmann equation. Our general sentiment was that two-term data are well acceptable in fluid modelling of streamers, though additional testing is required for gases with large anisotropy of the velocity distribution function in velocity space.

\section{Acknowledgments}

It is a pleasure to acknowledge very helpful discussions with Robert Robson, Willem Hundsdorfer and Chao Li. Furthermore, SD and AM acknowledge support from STW-projects 10118 and 10751, part of the Netherlands's Organization for Scientific Research (NWO). SD has also been supported by the MNTR, Serbia, under the contract number ON171037. RDW is supported by the Australian Research Council and the Centre for Antimatter-Matter Studies, Australia. 


\section{Appendix. Alternative forms of the high-order model}

To make contact with previous work $[46,51,52,54]$, we here present different forms of equations (29)-(32). When we evaluate the averages over the velocities $c$ in these equations and perform a considerable amount of algebra, we find

$\frac{\partial n}{\partial t}+\nabla \cdot n \boldsymbol{v}=C_{1}$,

$\frac{\partial}{\partial t}(n m \boldsymbol{v})+\nabla \cdot(n m \boldsymbol{v} \boldsymbol{v})+\nabla \cdot \boldsymbol{P}-n e \boldsymbol{E}=C_{m c}$,

$\frac{\partial}{\partial t}\left[n\left(\frac{1}{2} m v^{2}+\frac{3}{2} k T\right)\right]$

$+\nabla \cdot\left[\left(\frac{1}{2} m v^{2}+\frac{3}{2} k T\right) n \boldsymbol{v}+\boldsymbol{P} \cdot \boldsymbol{v}+\boldsymbol{q}\right]-n e \boldsymbol{E} \cdot \boldsymbol{v}=C_{\frac{1}{2} m c^{2}}$,

$$
\begin{aligned}
\frac{\partial}{\partial t}[ & \left.\left(\frac{1}{2} m v^{2}+\frac{3}{2} k T\right) n \boldsymbol{v}+\boldsymbol{P} \cdot \boldsymbol{v}+\boldsymbol{q}\right] \\
& +\nabla \cdot\left[2 \boldsymbol{v}(\boldsymbol{P} \cdot \boldsymbol{v})+2 \boldsymbol{v} \boldsymbol{q}+\frac{1}{2} v^{2} \boldsymbol{P}+\boldsymbol{Q}: \boldsymbol{v}+\boldsymbol{S}\right. \\
& \left.+\left(\frac{1}{2} m v^{2}+\frac{3}{2} k T\right) n \boldsymbol{v} \boldsymbol{v}\right]-\frac{e}{m}\left(\frac{1}{2} m \boldsymbol{v}^{2}+\frac{3}{2} k T\right) n \boldsymbol{E} \\
& -e n(\boldsymbol{v} \boldsymbol{v}) \cdot \boldsymbol{E}-\frac{e n}{m} \boldsymbol{P} \cdot \boldsymbol{E}=C_{\frac{1}{2} m c^{2} c}
\end{aligned}
$$

where $\boldsymbol{P}$ is the pressure tensor, $\boldsymbol{q}=\frac{1}{2} n m\left\langle(\boldsymbol{c}-\boldsymbol{v})^{2}(\boldsymbol{c}-\boldsymbol{v})\right\rangle$ is the heat flux vector, $S=\frac{1}{2} n m\left\langle(c-v)^{2}(c-v)(c-v)\right\rangle$ is the high-order pressure tensor and $\boldsymbol{Q}=n m\langle(\boldsymbol{c}-\boldsymbol{v})(\boldsymbol{c}-\boldsymbol{v})(\boldsymbol{c}-\boldsymbol{v})\rangle$ is the high-order heat flux tensor. The pressure tensors $\boldsymbol{P}$ and $\boldsymbol{S}$ are the second-order tensors while the high-order energy flux tensor $Q$ is a third-order tensor.

Using the continuity equation (A.1), the momentum balance equation (A.2) can be written as

$n m\left[\frac{\partial \boldsymbol{v}}{\partial t}+(\boldsymbol{v} \cdot \nabla) \boldsymbol{v}\right]-\nabla \cdot \boldsymbol{P}-e n \boldsymbol{E}=C_{m c}-m \boldsymbol{v} C_{1}$.

This equation is equivalent to equation (14) and can be used to exclude the energy of macroscopic motion from the energy (A.3) and energy flux (A.4) balance equations, respectively. For this purpose, we multiply momentum balance equations (A.2) and (A.5) by $\boldsymbol{v}$ and after addition of one of the resulting equations to another one, we obtain

$$
\begin{aligned}
& \frac{\partial}{\partial t}\left(\frac{1}{2} n m v^{2}\right)+\nabla \cdot\left(\frac{1}{2} n m v^{2} \boldsymbol{v}\right)+(\nabla \cdot P) \cdot \boldsymbol{v} \\
& \quad=\boldsymbol{v} C_{m c}-\frac{1}{2} v^{2} C_{1} .
\end{aligned}
$$

This is the balance equation for the energy of macroscopic motion. Taking this equation back into the energy and energy flux balance equations, we obtain an alternative and more compact form of the fluid equations:

$$
\begin{aligned}
& \frac{\partial n}{\partial t}+\nabla \cdot n \boldsymbol{v}=C_{1}, \\
& n m \frac{\mathrm{d} \boldsymbol{v}}{\mathrm{d} t}+\nabla \cdot \boldsymbol{P}-e n \boldsymbol{E}=C_{m \boldsymbol{v}_{\mathrm{r}}},
\end{aligned}
$$

$$
\begin{aligned}
& \frac{\mathrm{d}}{\mathrm{d} t}\left(\frac{3}{2} p\right)+\frac{3}{2} p(\nabla \cdot \boldsymbol{v})+\nabla \cdot \boldsymbol{q}+\boldsymbol{P}: \nabla \boldsymbol{v}=C_{\frac{1}{2} m v_{\mathrm{r}}^{2}}, \\
& \frac{\mathrm{d} \boldsymbol{q}}{\mathrm{d} t}+\boldsymbol{q} \cdot \nabla \boldsymbol{v}+\boldsymbol{q}(\nabla \cdot \boldsymbol{v})+\boldsymbol{Q}: \nabla \boldsymbol{v}+\nabla \cdot \boldsymbol{S} \\
& \quad+\left(\frac{\mathrm{d} \boldsymbol{v}}{\mathrm{d} t}-\frac{e}{m} \boldsymbol{E}\right)\left(\boldsymbol{\tau}+\frac{5}{2} p \boldsymbol{I}\right)=C_{\frac{1}{2} m v_{\mathrm{r}}^{2} \boldsymbol{v}_{\mathrm{r}},}
\end{aligned}
$$

where $\boldsymbol{v}_{\mathrm{r}}=\boldsymbol{c}-\boldsymbol{v}$ is the random velocity. The pressure $p$ is defined as one-third the trace of the pressure tensor

$$
p=\frac{1}{3} \sum_{i} P_{i i}=\frac{m}{3} \int(c-v)^{2} f \mathrm{~d} c,
$$

while $\tau$ is the stress tensor

$$
\tau=\boldsymbol{P}-p \boldsymbol{I},
$$

where $\boldsymbol{I}$ is the unity tensor (diagonal elements equal to unity). In equations (A.8)-(A.10), the convective time derivative (13) has been utilized. The collisional terms on the right-hand side of the balance equations (A.8)-(A.10) are given by

$$
\begin{aligned}
C_{m v_{\mathrm{r}}} & =-\int m v_{\mathrm{r}} J(f) \mathrm{d} v_{\mathrm{r}}, \\
C_{\frac{1}{2} m v_{\mathrm{r}}^{2}} & =-\int \frac{1}{2} m v_{\mathrm{r}}^{2} J(f) \mathrm{d} v_{\mathrm{r}}, \\
C_{\frac{1}{2} m v_{\mathrm{r}}^{2} v_{\mathrm{r}}} & =-\int \frac{1}{2} m v_{\mathrm{r}}^{2} \boldsymbol{v}_{\mathrm{r}} J(f) \mathrm{d} \boldsymbol{v}_{\mathrm{r}},
\end{aligned}
$$

where $J(f)$ is the collision operator and $f$ is the distribution function of charged particles. It should be noted that balance equations (A.7)-(A.10) can be derived directly from the Boltzmann equation. In such a case, however, the Boltzmann equation must be transformed into an equation for $\boldsymbol{v}_{\mathrm{r}}$ before the velocity moments are taken.

\section{References}

[1] Pasko V 2006 Sprites, Elves and Intense Lightning Discharges ed M Fullekrug (Berlin: Springer) p 253

[2] Ebert U, Nijdam S, Li C, Luque A, Briels T M P and van Veldhuizen E M 2010 J. Geophys. Res. 115 A00E43

[3] Luque A and Gordillo-Vazquez F J 2012 Nature Geosci. 522

[4] Ebert U and Sentman D D 2008 J. Phys. D: Appl. Phys. 41230301

[5] Winands G J J, Liu Z, Pemen A J M, van Heesch E J M and Yan K 2008 J. Phys. D: Appl. Phys. 41234001

[6] van Heesch E J M, Winands G J J and Pemen A J M 2008 J. Phys. D: Appl. Phys. 41234015

[7] Seeger M, Niemeyer L and Bujotzek M 2009 J. Phys. D: Appl. Phys. 42185205

[8] van Veldhuizen E M 2000 Electrical Discharges for Environmental Purposes: Fundamentals and Applications (Huntington, NY: Nova Science)

[9] Grabowski L R, van Veldhuizen E M, Pemen A J and Rutgers W R 2006 Plasma Chem. Plasma Process. 263

[10] Babayan S, Jeong J, Tu V, Park J, Selwyn G and Hicks R 1998 Plasma Sources Sci. Technol. 7286

[11] Jiang N, Ji A and Cao Z 2009 J. Appl. Phys. 106013308

[12] Naidis G V 2010 J. Phys. D: Appl. Phys. 43402001

[13] Naidis G 2011 J. Phys. D: Appl. Phys. 44215203

[14] Yousfi M, Eichwald O, Merbahi N and Jomaa N 2012 Plasma Sources Sci. Technol. 21045003 
[15] Xiong Z and Kushner M J 2012 Plasma Sources Sci. Technol. 21034001

[16] Boeuf J-P, Yang L L and Pitchford L C 2013 J. Phys. D: Appl. Phys. 46015201

[17] Naidis G V 2005 J. Phys. D: Appl. Phys. 383889

[18] Chaudhury B, Boeuf J P, Zhu G Q and Pascal O 2011 J. Appl. Phys. 110113306

[19] Fonte P 2002 IEEE Trans. Nucl. Sci. 49881

[20] Khorashad L K, Eskandari M and Moshaii A 2011 Nucl. Instrum. Methods A 628470

[21] Denni U, Felici G, Frani M A, Mengucci A, Papalino G, Spinetti M and Paolini A 2011 Nucl. Instrum. Methods A $\mathbf{6 4 0} 76$

[22] Luque A and Ebert U 2012 J. Comput. Phys. 231904

[23] Li C, Ebert U and Hundsdorfer W 2010 J. Comput. Phys. 229200

[24] Li C, Ebert U and Hundsdorfer W 2012 J. Comput. Phys. 2311020

[25] Li C, Teunissen J, Nool M, Willem Hundsdorfer and Ebert U 2012 Plasma Sources Sci. Technol. 21055019

[26] Gogolides E and Sawin H H 1992 J. Appl. Phys. 723971

[27] Crompton R W 1994 Adv. At. Mol. Opt. Phys. 3297

[28] Petrović Z Lj, Šuvakov M, Nikitović Ž, Dujko S, Šašić O, Jovanović J, Malović G and Stojanović V 2007 Plasma Sources Sci. Technol. 16 S1

[29] Petrović Z Lj, Dujko S, Marić D, Malović G, Nikitović Ž, Šašić O, Jovanović J, Stojanović V and Radmilović-Radjenovic M 2009 J. Phys. D: Appl. Phys. 31194002

[30] Raju G G 2012 Gaseous Electronics: Tables, Atoms and Molecules (Boca Raton, FL/London: CRC Press/Taylor and Francis)

[31] Anzai K et al 2012 Eur. Phys. J. D 6636

[32] Li C, Brok W J M, Ebert U and van der Mullen J J A M 2007 J. Appl. Phys. 101123305

[33] Aleksandrov N and Kochetov I 1996 J. Phys. D: Appl. Phys. 291476

[34] Naidis G V 1997 Tech. Phys. Lett. 23493

[35] Li C, Ebert U, Brok W J M and Hundsdorfer W 2008 J. Phys. D: Appl. Phys. 41032005

[36] White R D, Robson R E, Dujko S, Nicoletopoulos P and Li B 2009 J. Phys. D: Appl. Phys. 42194001

[37] Dujko S, White R D, Petrović Z Lj and Robson R E 2010 Phys. Rev. E 81046403

[38] Hagelaar G J M and Pitchford L C 2005 Plasma Sources Sci. Technol. 14722

[39] Robson R E and Ness K F 1986 Phys. Rev. A 332068

[40] Ness K F and Robson R E 1986 Phys. Rev. A 342185

[41] Ebert U, van Saarloos W and Caroli C 1996 Phys. Rev. Lett. 774178

[42] Ebert U, van Saarloos W and Caroli C 1997 Phys. Rev. E 551530

[43] Markosyan A, Dujko S and Ebert U 2013 High order fluid model for streamer discharges: II. Numerical solution and investigation of planar fronts J. Phys. D: Appl. Phys. 46475203

[44] Robson R E, White R D and Petrović Z Lj 2005 Rev. Mod. Phys. 771303
[45] Robson R E, Nicoletopoulos P, Li B and White R D 2008 Plasma Sources Sci. Technol. 17024020

[46] Kanzari Z, Yousfi M and Hamani A 1998 J. Appl. Phys. 844161

[47] Robson R E 1984 Aust. J. Phys. 3735

[48] Mason E A and McDaniel E W 1988 The Transport Properties of Ions in Gases (New York: Wiley)

[49] Robson R E 1986 J. Chem. Phys. 854486

[50] Abbas I and Bayle P 1980 J. Phys. D: Appl. Phys. 131055

[51] Abbas I and Bayle P 1981 J. Phys. D: Appl. Phys. 14649

[52] Bayle P and Cornebois B 1985 Phys. Rev. A 311046

[53] Guo J M and Wu C H J 1993 IEEE Trans. Plasma Sci. 21684

[54] Eichwald O, Ducasse O, Merbahi N, Yousfi M and Dubois D 2006 J. Phys. D: Appl. Phys. 3999

[55] Nicoletopoulos P and Robson R E 2008 Phys. Rev. Lett. 100124502

[56] Sobota A, Manders F, van Veldhuizen E M, van Dijk J and Haverlag M 2010 IEEE Trans. Plasma Sci. 382289

[57] Davoudabadi M, Shrimpton J S and Mashayek F 2009 J. Comput. Phys. 2282468

[58] Sima W, Peng Q, Yang Q, Yuan T and Shi J 2012 IEEE Trans. Dielectr. Electr. Insul. 19660

[59] Robson R E and Ness K F 1988 J. Chem. Phys. 894815

[60] Vrhovac S B and Petrović Z Lj 1996 Phys. Rev. E 534012

[61] Li B, White R D and Robson R E 2001 Ann. Phys. 292179

[62] Dujko S, Ebert U, White R D and Petrović Z Lj 2011 Japan. J. Appl. Phys. 50 08JC01

[63] Dujko S, White R D, Raspopović Z M and Petrović Z Lj 2012 Nucl. Instrum. Methods B 27984

[64] Sakai Y, Tagashira H and Sakamoto S 1977 J. Phys. D: Appl. Phys. 101035

[65] Robson R E 1995 Aust. J. Phys. 48347

[66] Dujko S, White R D and Petrović Z Lj 2008 J. Phys. D: Appl. Phys. 41245205

[67] Robson R E 1991 Aust. J. Phys. 44685

[68] Boeuf J P and Pitchford L P 1995 Phys. Rev. E 511376

[69] Kumar K, Skullerud H and Robson R E 1980 Aust. J. Phys. 33343

[70] Stojanović V D and Petrović Z Lj 1998 J. Phys. D: Appl. Phys. 31834

[71] Boltzmann L 1872 Wein. Ber. 66275 (in German)

[72] Wang-Chang C S, Uhlenbeck G E and De Boer J 1964 Studies in Statistical Mechanics vol II, ed J De Boer and G E Uhlenbeck (New York: Wiley) p 241

[73] Huxley L G and Crompton R W 1974 The Diffusion and Drift of Electrons in Gases (New York: Wiley)

[74] Cosby P C 1993 J. Chem. Phys. 989544

[75] Phelps A V and Pitchford L C 1985 JILA Report no 25

[76] Hundsdorfer W and Verwer J G 2003 Numerical Solution of Time-Dependent Advection-Diffusion-Reaction Equations (Series in Compuational Mathematics vol 33) (Berlin: Springer)

[77] Raether H 1964 Electron Avalanches and Breakdown in Gases (London: Butterworths)

[78] Montijn C and Ebert U 2006 J. Phys. D: Appl. Phys. 392979

[79] Montijn C, Hundsdorfer W and Ebert U 2006 J. Comput. Phys. 219801

[80] Ebert U and van Saarloos W 2000 Phys. Rep. 337139 